\title{
COHOMOLOGIE COHÉRENTE ET REPRÉSENTATIONS GALOISIENNES
}

\author{
par \\ Vincent Pilloni et Benoît Stroh
}

\begin{abstract}
Résumé. - Dans cet article, on montre comment les idées introduites dans l'article [S4] s'appliquent à l'étude de la cohomologie cohérente des variétés de Siegel, et plus généralement des variétés de Shimura de type Hodge. Le résultat principal affirme que les classes de cohomologie cohérente supérieure sont des limites $p$-adique de formes modulaires cuspidales. Ceci permet dans certains cas d'associer des représentations Galoisiennes à des formes automorphes apparaissant dans la cohomologie cohérente.
\end{abstract}

\section{Table des matières}

1. Modèle étrange des variétés de Siegel....................... 3

2. Le cas Hodge........................................ 10

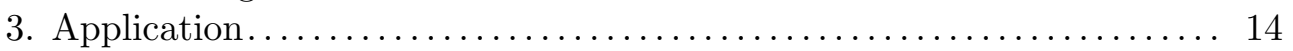

Appendice A. Compactifications.......................... 21

Références.......................................... 30

Soit $p$ un nombre premier, $G$ un groupe réductif sur $\mathbb{Q}, K=K_{p} K^{p}$ un sous-groupe compact ouvert de $G\left(\mathbb{A}_{f}\right)$. On fixe une donnée de Shimura pour le groupe $G$ et on suppose que la variété de Shimura associée $X_{G}(K)$ est de type Hodge. Elle est définie sur un corps de nombre $E$. Fixons un plongement $E \hookrightarrow \mathbb{C}_{p}$ et voyons $X_{G}(K)$ comme un schéma sur $\mathbb{C}_{p}$. Soit $X_{G}(K)^{\text {tor }}$ une compactification toroïdale et soit $\omega^{\kappa}$ un faisceau cohérent automorphe de poids $\kappa$ sur $X_{G}(K)^{t o r}$. Soit aussi $D$ le diviseur $X_{G}(K)^{t o r} \backslash X_{G}(K)$. Soit $\mathbb{T}$ l'algèbre de Hecke à coefficient dans $\mathbb{Z}_{p}$, engendrée par les opérateurs de niveau premier à $K$ et à $p$. Elle agit sur les groupes de cohomologie cohérente $\mathrm{H}^{i}\left(X_{G}(K)^{t o r}, \omega^{\kappa}\right)$ ou $\mathrm{H}^{i}\left(X_{G}(K)^{\text {tor }}, \omega^{\kappa}(-D)\right)$. Soit $\mathbb{T}^{p-a d}$ l'algèbre de Hecke $p$-adique de niveau $K^{p}$. C'est la limite projective sur les niveaux $K^{\prime}=K_{p}^{\prime} K^{p}$ et les ensembles finis de poids $\left\{\kappa_{1}, \cdots, \kappa_{r}\right\}$ des $\mathbb{Z}_{p^{-}}$-algèbres finies :

$$
\mathbb{T}\left(K^{\prime},\left\{\kappa_{1}, \cdots, \kappa_{r}\right\}\right)=\operatorname{Im}\left(\mathbb{T} \rightarrow \operatorname{End}\left(\mathrm{H}^{0}\left(X_{G}\left(K^{\prime}\right)^{t o r}, \omega^{\kappa_{1}}(-D) \oplus \cdots \oplus \omega^{\kappa_{r}}(-D)\right)\right) .\right.
$$

L'algèbre $\mathbb{T}^{p-a d}$ est munie de la topologie faible qui rend continues les applications $\mathbb{T}^{p-a d} \rightarrow$ $\mathbb{T}\left(K^{\prime},\left\{\kappa_{1}, \cdots, \kappa_{r}\right\}\right)$ où $\mathbb{T}\left(K^{\prime},\left\{\kappa_{1}, \cdots, \kappa_{r}\right\}\right)$ est muni de la topologie $p$-adique.

Théorème 0.1. - L'action de $\mathbb{T}$ agissant sur un groupe de cohomologie $\mathrm{H}^{i}\left(X_{G}(K)^{t o r}, \omega^{\kappa}\right)$ ou $\mathrm{H}^{i}\left(X_{G}(K)^{t o r}, \omega^{\kappa}(-D)\right)$ se factorise en une action continue de $\mathbb{T}^{p-a d}$. 
Remarque 0.2. - Soit $\lambda$ un système de valeurs propres pour l'action de $\mathbb{T}$ sur un groupe de cohomologie cohérente. Si on sait associer à toute forme holomorphe cuspidale propre de poids régulier un système de représentations galoisiennes, alors on peut associer à $\lambda$ un système de représentations galoisiennes (voir la section 3.11) qui vérifiera la compatibilité local-global aux places non-ramifiées.

Remarque 0.3. - Dans l'article nous introduisons des modèles entiers sur $\mathcal{O}_{\mathbb{C}_{p}}$ pour les variétés de Shimura et les faisceaux $\omega^{\kappa}$ et nous déduisons le théorème du résultat plus général que les représentations de $\mathbb{T}$ sur les groupes de cohomologie de ces modèles entiers se factorisent par $\mathbb{T}^{p-a d}$ (voir par exemple la remarque 3.10). Nos résultats valent en particulier pour les classes de torsion de certains modèles entiers.

La démonstration repose sur l'introduction [S4] de modèles entiers $p$-adique étranges des compactifications minimales des variétés de Shimura et sur l'existence, dès que le niveau est suffisamment ramifié en $p$, de formes modulaires de torsion, "les classes de Hodge-Tate", qui commutent à l'action de l'algèbre de Hecke de niveau premier à $p$. Ces formes jouent un rôle semblable à l'invariant de Hasse. Leur grand avantage est de former un système linéaire sans point base du faisceau de Hodge convenablement modifié, lequel est ample sur le modèle étrange. Un calcul à la Chech de la cohomologie cohérente permet de représenter les classes de cohomologie par des sections définies sur des ouverts de nonannulation de certaines classes de Hodge-Tate, qu'on peut approximer $p$-adiquement, en respectant l'action de Hecke, par des formes modulaires classiques.

Dans la première partie de cet article, nous étudions la relation entre les modèles entiers de Kottwitz des compactifications toroïdales et minimales des variétés de Siegel et les modèles étranges de la compactification minimale. En effet, les faisceaux cohérents automorphes sont définit sur les modèles de Kottwitz des compactifications toroïdales, tandis que pour la démonstration du théorème il est crucial de travailler sur les modèles étranges de la compactification minimale. On introduit une modification commune du modèle étrange et du modèle de Kottwitz qui permet de faire le pont. Ceci nous amène à construire l'application des périodes de Hodge-Tate au niveau formel sur les compactifications minimales et toroïdales, ce qui offre une alternative à certains arguments de la section 3 de $[\mathbf{S} 4]$.

Dans la seconde partie nous passons des variétés de Siegel aux variétés de type Hodge, puis dans la troisième partie nous démontrons le théorème.

Dans l'appendice, nous décrivons les compactifications toroïdales des modèles entiers des variétés de Siegel que nous utilisons (qui ont un niveau principal $p^{n}$ ). Nous en profitons pour démontrer le théorème suivant :

Théorème 0.4. - Soit $\mathcal{X}\left(p^{n}\right)^{\text {tor }}$ une compactification toroidale de la variété de Siegel rigide de niveau plein $p^{n}$ en $p$. Alors il existe un espace perfectoide $\mathcal{X}\left(p^{\infty}\right)^{\text {tor-mod }} \sim$ $\lim _{n} \mathcal{X}\left(p^{n}\right)^{\text {tor }}$.

Le symbole $\sim$ est celui de la définition 3.15 de $[\mathbf{S W}]$. Il signifie qu'on a des morphismes compatibles $\mathcal{X}\left(p^{\infty}\right)^{\text {tor-mod }} \rightarrow \mathcal{X}\left(p^{n}\right)^{\text {tor }}$ qui induisent un isomorphisme d'espaces topologiques $\left|\mathcal{X}\left(p^{\infty}\right)^{\text {tor-mod }}\right| \simeq \lim _{n}\left|\mathcal{X}\left(p^{n}\right)^{\text {tor }}\right|$ et qu'on possède un recouvrement par des affinoides $\mathcal{X}\left(p^{\infty}\right)^{\text {tor-mod }}=\cup_{i} U_{i}$ tel que

$$
\mathscr{O}_{\mathcal{X}\left(p^{\infty}\right)^{\text {tor-mod }}}\left(U_{i}\right)=\left(\operatorname{colim}_{n} \widehat{\mathscr{O}_{\mathcal{X}\left(p^{n}\right)^{\text {tor }}}}\left(U_{i}\right)\right) .
$$

Dans cette dernière formule, la complétion sur le membre de droite est par rapport à la norme spectrale. Dans notre cas, le recouvrement $\cup_{i} U_{i}$ est d'ailleurs assez explicite. 
Nous remercions W. Goldring de nous avoir initié à la problématique des représentations automorphes limites de séries discrètes à l'infini et $\mathrm{P}$. Scholze pour d'utiles discussions. Signalons que G. Boxer, D. Geraghty et W. Goldring ont obtenu, par des méthodes différentes, des résultats du même type que ceux du théorème 0.1.

\section{Modèle étrange des variétés de Siegel}

Dans cette partie, nous rappelons la construction due à P. Scholze d'un modèle formel de la compactification minimale des variétés de Siegel de niveau $p^{n}$. Une puissance convenable du faisceau de Hodge modifié définit un faisceau inversible ample sur ces modèles, et l'application de Hodge-Tate permet de construire un système linéaire sans point base de formes modulaires de torsion qui commutent à l'action des opérateurs de Hecke de niveau premier à $p$.

1.1. Construction de schémas formels par normalisation. - Soit $\mathfrak{Z}$ un schéma formel topologiquement de type fini sur $\operatorname{Spf} \mathcal{O}_{\mathbb{C}_{p}}$ et $\mathcal{Z}$ sa fibre générique au sens de Raynaud. On suppose que $\mathcal{Z}$ est réduit. On dit que $\mathfrak{Z}$ est intégralement clos dans $\mathcal{Z}$ si pour tout ouvert affine $\mathfrak{U}$ de $\mathfrak{Z}$, de fibre générique $\mathcal{U}$, l'une des propriétés équivalentes est satisfaite :

- $\mathscr{O}_{\mathfrak{Z}}(\mathfrak{U})$ est intégralement clos dans $\mathscr{O}_{\mathfrak{Z}}(\mathfrak{U})[1 / p]=\mathscr{O}_{\mathcal{Z}}(\mathcal{U})$,

- $\mathscr{O}_{\mathfrak{Z}}(\mathfrak{U})$ est la sous-algèbre des fonctions à puissance bornée de $\mathscr{O}_{\mathcal{Z}}(\mathcal{U})$,

- $\mathscr{O}_{\mathfrak{Z}}(\mathfrak{U})$ est la sous-algèbre des fonctions de norme spectrale inférieure à 1 de $\mathscr{O}_{\mathcal{Z}}(\mathcal{U})$.

Soit $\mathfrak{Z}$ un schéma formel topologiquement de type fini sur $\operatorname{Spf} \mathcal{O}_{\mathbb{C}_{p}}$ de fibre générique $\mathcal{Z}$ réduite. Soit $\mathcal{T}$ un espace rigide réduit muni d'un morphisme fini $g: \mathcal{T} \rightarrow \mathcal{Z}$.

Proposition 1.2. - Il existe un unique schéma formel $\mathfrak{T}$ de fibre générique $\mathcal{T}$, intégralement clos dans $\mathcal{T}$ muni d'un morphisme fini $\mathfrak{g}: \mathfrak{T} \rightarrow \mathfrak{Z}$ s'insérant dans le diagramme commutatif :

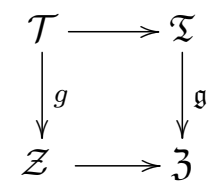

Le schéma formel $\mathfrak{T}$ est appelé normalisé de $\mathfrak{Z}$ dans $\mathcal{T}$.

Démonstration. Soit $\mathscr{O}_{\mathcal{T}}^{0}$ le sous-faisceau de $\mathscr{O}_{\mathcal{T}}$ des sections à puissance bornée. Commençons par définir un faisceau $\mathscr{F}$ de $\mathscr{O}_{\mathfrak{Z}}$-modules par la règle suivante. Soit $\mathfrak{U}$ un ouvert de $\mathfrak{Z}$ de fibre générique $\mathcal{U}$. On pose

$$
\mathscr{F}(\mathfrak{U})=\mathscr{O}_{\mathcal{T}}^{0}\left(g^{-1}(\mathcal{U})\right) .
$$

Nous allons à présent vérifier que le faisceau $\mathscr{F}$ est un faisceau cohérent de $\mathscr{O}_{3}$-algèbres. On définira alors $\mathfrak{T}$ comme le spectre formel, relativement à $\mathfrak{Z}$, de $\mathscr{F}$.

Soit Spf $R$ un ouvert formel de $\mathfrak{Z}$. Soit $R^{0}$ la sous-algèbre des fonctions à puissances bornées de $R[1 / p]$. C'est une $R$-algèbre finie. Soit $\operatorname{Spa}\left(S, S^{0}\right)$ l'image inverse de Spa $\left(R[1 / p], R^{0}\right)$ dans $\mathcal{T}$. D'après $[\mathbf{B G R}]$, coro. 5, p. $251, S^{0}$ est une $R^{0}$-algèbre finie et donc une $R$-algèbre finie. Soit $f \in R$ et soit Spf $R<f^{-1}>$ l'ouvert $f \neq 0$. Il nous faut démontrer que le morphisme de localisation :

$$
\mathscr{F}(R) \otimes_{R} R<f^{-1}>\longrightarrow \mathscr{F}\left(R<f^{-1}>\right)
$$


est un isomorphisme. Soit Spa $\left(S<f^{-1}>,\left(S<f^{-1}>\right)^{0}\right)$ l'ouvert $|f|=1$ dans Spa $\left(S, S^{0}\right)$. Il s'agit donc de voir que $S^{0}<f^{-1}>=\left(S<f^{-1}>\right)^{0}$. L'inclusion $\subset$ est évidente. De plus, $S^{0}<f^{-1}>$ est ouvert dans $\left(S<f^{-1}>\right)^{0}$. Pour montrer l'inclusion réciproque, il suffit de voir que $S^{0}\left[f^{-1}\right]=S\left[f^{-1}\right] \cap\left(S<f^{-1}>\right)^{0}$ ou, de façon équivalente, que $S \cap\left(S<f^{-1}>\right)^{0} \subset S^{0}\left[f^{-1}\right]$. Soit $s \in S$ une fonction qui est bornée par 1 sur l'ouvert $|f|=1$. Pour toute valuation $x$ de rang 1 sur Spa $\left(S, S^{0}\right)$, on a $\left|f^{n} s\right|_{x} \leq 1$ si $x \in|f|=1$ et $\lim _{n \rightarrow \infty}\left|f^{n} s\right|_{x} \rightarrow 0$ si $x \notin|f|=1$. Soit $\left\{x_{1}, \ldots, x_{m}\right\}$ le bord de Shilov de Spa $\left(S, S^{0}\right)$. Alors, pour $n$ suffisament grand, on a $\left|f^{n} s\right|_{x_{i}} \leq 1$ et donc $f^{n} s \in S^{0}$.

1.3. Variétés de Siegel de niveau $p^{n}$. - Dans cette partie, nous noterons $G=$ $\mathrm{GSp}_{2 g} / \mathbb{Q}$ le groupe des similitudes symplectiques usuel, $\mathbb{A}_{f}^{p}$ l'anneau des adèles finies hors $p$ de $\mathbb{Q}$ et $K^{p} \subset G\left(\mathbb{A}_{f}^{p}\right)$ un sous-groupe ouvert compact tel que $K^{p} G\left(\mathbb{Z}_{p}\right)$ soit net. Le sousgroupe $K^{p}$ ne jouera aucun autre rôle que d'assurer la représentabilité des variétés de Siegel. On note $X$ la variété de Siegel de genre $g$ définie sur $\mathcal{O}_{\mathbb{C}_{p}}$ de niveau $K^{p}$ hors $p$ et de niveau hyperspécial en $p$. On note $X^{\star}$ sa compactification minimale $[\mathbf{F C}], X^{\text {tor }}$ la compactification toroïdale $[\mathbf{F C}]$ associée à un choix combinatoire $\mathcal{S}$ décrit dans l'appendice A.1 et $A \rightarrow \mathfrak{X}^{\text {tor }}$ le schéma semi-abélien qui étend le schéma abélien universel. Soit $\mathfrak{X}$ la complétion formelle $p$-adique de $X$ et $\mathfrak{X}^{\text {tor }}$ la complétion formelle $p$-adique de $X^{\text {tor }}$. Soit $\mathcal{X}$ la fibre générique de $\mathfrak{X}$ et $\mathcal{X}^{\text {tor }}$ la fibre générique de $\mathfrak{X}^{\text {tor }}$. Notons $\mathcal{X}\left(p^{n}\right)$ la variété rigide, finie étale galoisienne de groupe $\operatorname{GSp}_{2 g}\left(\mathbb{Z} / p^{n} \mathbb{Z}\right)$ sur $\mathcal{X}$ qui paramètre une structure pleine de niveau $p^{n}$. Nous notons $\mathcal{X}\left(p^{n}\right)^{\text {tor }}$ la compactification toroïdale associée à $\mathcal{S}$. On a donc un morphisme fini $\mathcal{X}\left(p^{n}\right)^{\text {tor }} \rightarrow \mathcal{X}^{\text {tor }}$ et nous poserons $\mathfrak{X}\left(p^{n}\right)^{\text {tor }}$ la normalisation de $\mathfrak{X}^{\text {tor }}$ dans $\mathcal{X}\left(p^{n}\right)^{\text {tor }}$.

Notons $\mathfrak{X}^{\star}$ la complétion formelle $p$-adique de $X^{\star}$ qui est normale par construction. On a une application $\pi: \mathfrak{X}\left(p^{n}\right)^{\text {tor }} \rightarrow \mathfrak{X}^{\star}$. Définissons $\mathfrak{X}\left(p^{n}\right)^{\star}$ comme la factorisation de Stein du morphisme $\pi$. Autrement dit, $\mathfrak{X}\left(p^{n}\right)^{\star}$ est le schéma formel affine sur $\mathfrak{X}^{*}$ associé à

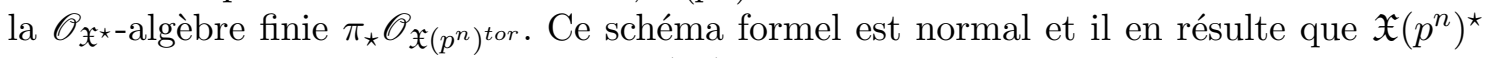
est aussi la normalisation de $\mathfrak{X}^{\star}$ dans $\mathcal{X}\left(p^{n}\right)^{\star}$, cette dernière désignant la compactification minimale de la variété rigide de niveau $p^{n}$.

1.4. Classes de Hodge-Tate. — L'application de Hodge-Tate permet de construire des formes modulaires de poids la représentation standard de $\mathrm{GL}_{g}$ modulo $p^{n}$ sur $\mathfrak{X}\left(p^{n}\right)^{\text {tor }}$. Ces formes modulaires commutent aux opérateurs de Hecke de niveau premier à $p$ et jouent un rôle semblable à l'invariant de Hasse. D'autre part, elles permettent de construire, en niveau $p^{\infty}$, une application des périodes de Hodge-Tate vers une grassmanienne.

Notons $e_{1}, \ldots, e_{2 g}$ la base canonique de $\mathbb{Z}_{p}^{2 g}$. Sur le schéma formel normal $\mathfrak{X}\left(p^{n}\right)$, on dispose d'un morphisme

$$
\left(\mathbb{Z} / p^{n} \mathbb{Z}\right)^{2 g} \rightarrow A\left[p^{n}\right]
$$

qui provient de l'isomorphisme tautologique en fibre générique. D'autre part, rappelons l'existence d'un morphisme de Hodge-Tate de faisceaux abéliens fppf

$$
H T: A\left[p^{n}\right] \longrightarrow \omega_{A} / p^{n} \omega_{A}
$$

où $\omega_{A}$ est le fibré vectoriel de rang $g$ sur $X$ formé des 1-formes différentielles invariantes sur $A$. En composant ces deux morphismes, on obtient des sections $H T\left(e_{1}\right), \ldots, H T\left(e_{2 g}\right) \in$ $\mathrm{H}^{0}\left(\mathfrak{X}\left(p^{n}\right), \omega_{A} / p^{n}\right)$. Le fasiceau $\omega_{A}$ se prolonge à la compactification toroïdale et son déterminant descend à la compactification minimale.

Proposition 1.5. - Les sections $H T\left(e_{1}\right), \ldots, H T\left(e_{2 g}\right)$ se prolongent en des sections de $\mathrm{H}^{0}\left(\mathfrak{X}\left(p^{n}\right)^{\text {tor }}, \omega_{A} / p^{n}\right)$. 
Démonstration. Le problème est local au bord pour la topologie étale. On utilise les notations de l'appendice (A). Soit $V^{\prime} \in \mathfrak{C}$. Sur $\mathfrak{M}_{V^{\prime}, n}$ on dispose du 1-motif principalement polarisé

$$
M_{V^{\prime}}=\left[V^{\perp} / V^{\prime} \rightarrow \tilde{G}\right] .
$$

L'application de Hodge-Tate $M_{V^{\prime}}\left[p^{n}\right] \rightarrow \omega_{\tilde{G}}$ est triviale sur la partie torique $T_{V^{\prime}}\left[p^{n}\right]$ et se factorise donc en une application

$$
\tilde{G}^{D}\left[p^{n}\right] \longrightarrow \omega_{\tilde{G}}
$$

Il en résulte que les classes $H T\left(e_{1}\right), \ldots, H T\left(e_{g}\right)$ appartiennent en fait à $\mathrm{H}^{0}\left(\mathfrak{B}_{V^{\prime}, n}, \omega_{\tilde{G}} / p^{n}\right)$. Par conséquent, elles sont définies sur $\mathrm{H}^{0}\left(\mathfrak{M}_{V^{\prime}, n, \sigma}, \omega_{\tilde{G}} / p^{n}\right)$ pour tout $\sigma \in \mathcal{S}$.

Remarque 1.6. - En fait, si $g \geq 2$, on pourrait démontrer que le principe de Koecher est valable dans ce contexte : pour tout groupe abélien $M$, on a un isomorphisme $\mathrm{H}^{0}\left(\mathfrak{X}\left(p^{n}\right)^{\text {tor }}, \omega_{A} \otimes M\right) \rightarrow \mathrm{H}^{0}\left(\mathfrak{X}\left(p^{n}\right), \omega_{A} \otimes M\right)$.

Posons $r=C_{2 g}^{g}$ le coefficient binômial. On note $e_{1}^{\prime}, \ldots, e_{r}^{\prime}$ une base de $\Lambda^{g} \mathbb{Z}_{p}^{2 g}$ obtenue en prenant des produits exterieurs de $e_{1}, \ldots, e_{2 g}$ et on note $s_{1}, \ldots, s_{r} \in \mathrm{H}^{0}\left(\mathfrak{X}\left(p^{n}\right)^{\text {tor }}\right.$, $\left.\operatorname{det} \omega_{A} / p^{n}\right)$ les éléments $\Lambda^{g} H T\left(e_{1}^{\prime}\right), \ldots, \Lambda^{g} H T\left(e_{r}^{\prime}\right)$.

Corollaire 1.7. - Les sections $s_{1}, \ldots, s_{r}$ proviennent par image inverse de sections notées de la même manière appartenant à

$$
\mathrm{H}^{0}\left(\mathfrak{X}\left(p^{n}\right)^{\star}, \operatorname{det} \omega_{A} / p^{n}\right)
$$

Démonstration. C'est la formule de projection et le corollaire A.10.

1.8. Modification du faisceau. - Rappelons le résultat clé suivant. Soit $R$ une $\mathcal{O}_{\mathbb{C}_{p}}{ }^{-}$ algèbre admissible normale (c'est à dire un quotient d'un anneau de série formelle restreint $\mathcal{O}_{\mathbb{C}_{p}}<X_{1}, \ldots, X_{n}>$ qui est plat comme $\mathcal{O}_{\mathbb{C}_{p}}$-algèbre et normal).

Théorème 1.9 ([F], thm. 7). - Supposons $p \geq 3$, soit $H \rightarrow$ Spec $R$ un groupe de Barsotti-Tate tronqué d'échelon $n$ et de hauteur $h$. On suppose que $H(R) \simeq\left(\mathbb{Z} / p^{n} \mathbb{Z}\right)^{h}$. Le conoyau de l'application de Hodge-Tate

$$
H T: H(R) \otimes_{\mathbb{Z}} R \rightarrow \omega_{H^{D}}
$$

est tué par l'idéal par tout élément de $\mathcal{O}_{\mathbb{C}_{p}}$ de valuation supérieure ou égale à $\frac{1}{p-1}$.

Remarque 1.10. - Fargues nous a indiqué que pour $p=2$, on a un résultat similaire avec $\frac{1}{p-1}$ remplacé par 2 . Bien que ce résultat ne soit pas rédigé pour l'instant, nous l'utiliserons dans ce qui suit lorsque $p=2$.

Remarque 1.11. - Dans la référence $[\mathbf{F}]$, le résultat est énoncé pour $R=\mathcal{O}_{\mathbb{C}_{p}}$. On obtient le cas général sans problème, en argumentant comme dans [AIP], prop. 4.2.2 par exemple.

On a établi, pour tout $n$, l'existence d'une application $H T:\left(\mathbb{Z} / p^{n} \mathbb{Z}\right)^{2 g} \otimes \mathscr{O}_{\mathfrak{X}\left(p^{n}\right)^{t o r}} \rightarrow$ $\omega_{A} / p^{n}$. On peut alors considérer le sous-faisceau $\omega_{A}^{\text {mod }} \subset \omega_{A}$ qui est défini comme l'image inverse de

$$
H T\left(\left(\mathbb{Z} / p^{n} \mathbb{Z}\right)^{2 g} \otimes \mathscr{O}_{\left.\mathfrak{X}\left(p^{n}\right)^{\text {tor }}\right)}\right.
$$

dans $\omega_{A}$. Pour tout $n$, on dispose également d'une application

$$
\Lambda^{g} H T:\left(\mathbb{Z} / p^{n} \mathbb{Z}\right)^{r} \otimes \mathscr{O}_{\mathfrak{X}\left(p^{n}\right)^{\star}} \longrightarrow \operatorname{det} \omega_{A} / p^{n} .
$$

On peut alors considérer le sous-faisceau $\operatorname{det} \omega_{A}^{\text {mod }} \subset \operatorname{det} \omega_{A}$ qui est défini comme l'image inverse de $\Lambda^{g} H T\left(\left(\mathbb{Z} / p^{n} \mathbb{Z}\right)^{r} \otimes \mathscr{O}_{\mathfrak{X}\left(p^{n}\right)^{\star}}\right)$ dans $\omega_{A}$. 
Lemme 1.12. - Les points suivants sont vérifiés :

1. les faisceaux $\omega_{A}^{\text {mod }}$ ne dépendent pas de $n \geq 1$ (resp. $n \geq 2$ si $p=2$ ).

2. les faisceaux $\operatorname{det} \omega_{A}^{\text {mod }}$ ne dépendent pas de $n \geq \frac{g}{p-1}$ (resp. $n \geq 2 g$ si $p=2$ ).

Démonstration. Supposons $p \geq 3$ dans cette démonstration, les arguments s'adaptent facilement au cas $p=2$ en changeant légèrement les bornes comme dans l'énoncé de la proposition. Comme le conoyau de l'application de Hodge-Tate est tué par $p$, on a $p \omega_{A} \subset \omega_{A}^{\text {mod }} \subset \omega_{A}$. Par conséquent, $\omega_{A}^{\text {mod }}$ est l'unique sous-faisceau de $\omega_{A}$ qui contient $p \omega_{A}$ et dont l'image dans $\omega_{A} \otimes \mathbb{Z} / p$ est engendré par l'image de l'application de Hodge-Tate. L'image de l'application de Hodge-Tate dans $\omega_{A} \otimes \mathbb{Z} / p$ ne dépend que du groupe de Barsotti-Tate tronqué au niveau 1. Le second point est analogue.

Les faisceaux $\omega_{A}^{\text {mod }}$ et $\operatorname{det} \omega_{A}^{\text {mod }}$ ne sont plus localement libres. Il est donc naturel d'introduire des modifications des espaces en jeu qui les rende localement libres.

Notation 1.1. - Soit $n_{0}$ le plus petit entier $>g /(p-1)\left(\right.$ resp $n_{0}=2 g+1$ si $\left.p=2\right)$.

Pour tout $n \geq n_{0}$, soit $I$ l'idéal de $\mathscr{O}_{\mathfrak{X}\left(p^{n}\right)^{\star}}$ engendré par des relèvements des coefficients de l'application

$$
\left(\mathbb{Z} / p^{n} \mathbb{Z}\right)^{r} \otimes \mathscr{O}_{\mathfrak{X}\left(p^{n}\right)^{\star}} \longrightarrow \operatorname{det} \omega_{A} / p^{n} .
$$

Remarquons que l'idéal $I$ est trivial en fibre générique et qu'il ne dépend pas des choix des relèvements. Soit $\tilde{\mathfrak{X}}\left(p^{n}\right)^{\star}$ l'éclatement de $\mathfrak{X}\left(p^{n}\right)^{\star}$ le long de l'idéal $I$. Par propriété universelle de l'éclatement, l'image inverse de det $\omega_{A}^{\text {mod }}$ sur $\tilde{\mathfrak{X}}\left(p^{n}\right)^{\star}$ est un idéal inversible. On définit enfin $\mathfrak{X}\left(p^{n}\right)^{\star-\bmod }$ comme la normalisation de $\tilde{\mathfrak{X}}\left(p^{n}\right)^{*}$ dans sa fibre générique.

Pour tout $n \geq n_{0}$, considérons les ideaux $I_{1}, \ldots, I_{g} \subset \mathscr{O}_{\mathfrak{X}\left(p^{n}\right)}$ tor engendrés par des relèvements des déterminants des mineurs de taille $g, \ldots, 1$ de l'application

$$
\left(\mathbb{Z} / p^{n} \mathbb{Z}\right)^{2 g} \otimes \mathscr{O}_{\mathfrak{X}\left(p^{n}\right)^{\text {tor }}} \longrightarrow \omega_{A} / p^{n} .
$$

Remarquons que ces idéaux sont inversibles en fibre générique et ne dépendent pas du choix des relèvements. On obtient alors $\tilde{\mathfrak{X}}\left(p^{n}\right)^{\text {tor }}$ en faisant la suite d'éclatement de $\mathfrak{X}\left(p^{n}\right)^{\text {tor }}$ en chacun de ces idéaux. Localement pour la topologie de Zariski sur $\tilde{\mathfrak{X}}\left(p^{n}\right)^{\text {tor }}$, l'application

$$
\left(\mathbb{Z} / p^{n} \mathbb{Z}\right)^{2 g} \otimes \mathscr{O}_{\tilde{\mathfrak{X}}\left(p^{n}\right)^{t o r}} \longrightarrow \omega_{A} / p^{n}
$$

peut être mise sous forme diagonale, et, comme précédemment, le faisceau $\omega_{A}^{\text {mod }}$ devient donc localement libre sur $\tilde{\mathfrak{X}}\left(p^{n}\right)^{\text {tor }}$. On définit enfin $\mathfrak{X}\left(p^{n}\right)^{\text {tor-mod }}$ comme la normalisation de $\tilde{\mathfrak{X}}\left(p^{n}\right)^{\text {tor }}$ dans sa fibre générique.

Proposition 1.13. - Les assertions suivantes sont vérifiées.

1. Pour tout $n \geq n_{0}$, on possède un schéma formel admissible normal $\mathfrak{X}\left(p^{n}\right)^{\star-m o d}$ et un morphisme projectif $f_{n}: \mathfrak{X}\left(p^{n}\right)^{\star-\text { mod }} \rightarrow \mathfrak{X}\left(p^{n}\right)^{\star}$ qui induit un isomorphisme d'espaces rigides, tel que le faisceau $f_{n}^{\star}\left(\operatorname{det} \omega_{A}^{\text {mod }}\right)$ soit localement libre de rang fini.

2. Pour tout $n \geq n_{0}$, on possède un schéma formel admissible normal $\mathfrak{X}\left(p^{n}\right)^{\text {tor-mod }}$ et un morphisme projectif $g_{n}: \mathfrak{X}\left(p^{n}\right)^{\text {tor-mod }} \rightarrow \mathfrak{X}\left(p^{n}\right)^{\text {tor }}$ qui induit un isomorphisme d'espaces rigides, tel que le faisceau $g_{n}^{\star}\left(\omega_{A}^{\text {mod }}\right)$ soit localement libre de rang fini.

3. Les constructions sont fonctorielles en $n$, en le niveau auxiliaire et en les morphismes de la compactification torö̈dale vers la compactification minimale. En particulier, pour tout $m \geq n \geq n_{0}$, on a des morphismes finis : $\mathfrak{X}\left(p^{m}\right)^{\text {tor-mod }} \rightarrow \mathfrak{X}\left(p^{n}\right)^{\text {tor-mod }}$ et $\mathfrak{X}\left(p^{m}\right)^{\star-\text { mod }} \rightarrow \mathfrak{X}\left(p^{n}\right)^{\star-\text { mod }}$, et pour tout $n \geq n_{0}$, on des diagrammes commutatifs : 


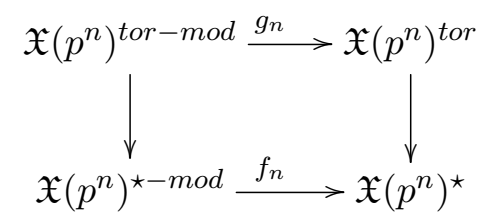

Démonstration. Le points 1 . et 2. résument la construction précédent la proposition. La fonctorialité ne pose pas de problème. Vérifions la finitude du morphisme $\mathfrak{X}\left(p^{m}\right)^{\text {tor-mod }} \rightarrow$ $\mathfrak{X}\left(p^{n}\right)^{\text {tor-mod }}$. Le morphisme $\tilde{\mathfrak{X}}\left(p^{m}\right)^{\text {tor }} \rightarrow \tilde{\mathfrak{X}}\left(p^{n}\right)^{\text {tor }}$ est affine et il est fini en fibre générique. On applique alors [BGR], coro. 5, p. 251.

1.14. Application des périodes formelle. - Soit $\mathfrak{X}\left(p^{\infty}\right)^{\star-\bmod }$ le schéma formel $p$ adique limite projective des $\mathfrak{X}\left(p^{n}\right)^{\star-m o d}$. Ce schéma est bien défini car les applications de transition sont affines pour $n \geq n_{0}$. De même, soit $\mathfrak{X}\left(p^{\infty}\right)^{\text {tor-mod }}$ le schéma formel limite projective des $\mathfrak{X}\left(p^{n}\right)^{\text {tor-mod }}$. On dispose sur $\mathfrak{X}\left(p^{\infty}\right)^{\star-\text { mod }}$ d'un faisceau inversible $\operatorname{det}\left(\omega_{A}^{\text {mod }}\right)$ et d'une application surjective

$$
\Lambda^{g} H T: \mathbb{Z}_{p}^{r} \otimes \mathscr{O}_{\mathfrak{X}\left(p^{\infty}\right)^{\star-m o d}} \longrightarrow \operatorname{det} \omega_{A}^{\text {mod }} .
$$

Il en résulte un morphisme

$$
\mathfrak{X}\left(p^{\infty}\right)^{\star-\bmod } \longrightarrow \mathbb{P}^{r-1} .
$$

Soit $\mathfrak{F} \mathfrak{L}$ la variété de drapeaux formelle $p$-adique qui paramètre des quotients Lagrangiens de dimension $g$ d'un module libre symplectique de dimension $2 g$. On a une immersion fermée de Plücker $\mathfrak{F} \mathfrak{L} \hookrightarrow \mathbb{P}^{r-1}$. On dispose alors aussi d'une application

$$
\mathfrak{X}\left(p^{\infty}\right)^{\text {tor-mod }} \longrightarrow \mathfrak{F} \mathfrak{L}
$$

obtenue grâce à la surjection

$$
H T: \mathbb{Z}_{p}^{2 g} \otimes \mathscr{O}_{\mathfrak{X}\left(p^{\infty}\right)^{\text {tor-mod }}} \longrightarrow \omega_{A}^{\text {mod }} .
$$

Proposition 1.15. - L'application $\mathfrak{X}\left(p^{\infty}\right)^{\star-m o d} \rightarrow \mathbb{P}^{r-1}$ se factorise en une application $\pi_{H T}: \mathfrak{X}\left(p^{\infty}\right)^{\star-\bmod } \rightarrow \mathfrak{F} \mathfrak{L}$.

Démonstration. Par construction, on dispose d'un diagramme commutatif :

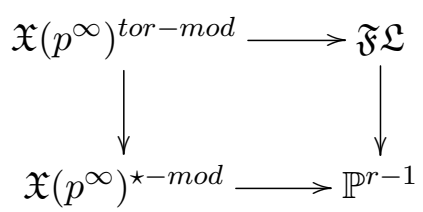

Pour démontrer la proposition, il suffit de vérifier que le morphisme $f: \mathfrak{X}\left(p^{\infty}\right)^{\text {tor-mod }} \rightarrow$ $\mathfrak{X}\left(p^{\infty}\right)^{\star-\text { mod }}$ est dominant.

Pour tout $m \geq n$, le morphisme

$$
\mathfrak{X}\left(p^{m}\right)^{\text {tor-mod }} \rightarrow \mathfrak{X}\left(p^{m}\right)^{\star-\text { mod }} \times_{\mathfrak{X}\left(p^{n}\right)^{\star-\text { mod }}} \mathfrak{X}\left(p^{n}\right)^{\text {tor-mod }}
$$

est surjectif. Il induit en effet, au niveau des espaces rigides, un isomorphisme sur le complémentaire du bord. Comme les applications de spécialisation sont surjectives, et que le bord est un fermé strict dans ces espaces, il en résulte que l'image du morphisme est dense. L'image est de plus fermée car le morphisme est projectif.

On en déduit alors, par passage à la limite, que le morphisme $f: \mathfrak{X}\left(p^{\infty}\right)^{\text {tor-mod }} \rightarrow$ $\mathfrak{X}\left(p^{\infty}\right)^{\star-\text { mod }}$ est surjectif. Vérifions que ceci entraîne bien que le morphisme $\mathscr{O}_{\mathfrak{X}\left(p^{\infty}\right)^{\star-m o d}} \rightarrow$ $f_{\star} \mathscr{O}_{\mathfrak{X}\left(p^{\infty}\right)^{\text {tor-mod }}}$ est injectif. Soit $\operatorname{Spf}\left(R_{n}\right)$ un ouvert affine de $\mathfrak{X}\left(p^{n}\right)^{\star-\text { mod }}$. Soit $\operatorname{Spf}\left(R_{k}\right)$ l'image inverse de cet ouvert dans $\mathfrak{X}\left(p^{k}\right)^{\star-m o d}$ et soit $R_{\infty}$ la complétion $p$-adique de la 
limite inductive des $R_{k}$. D'après le lemme A.15, on dispose d'une norme |.| sur $R_{\infty}$ telle que $\left|R_{\infty}\right|=\left|\mathcal{O}_{\mathbb{C}_{p}}\right|$ et telle que $R_{\infty}$ s'identifie aux éléments de norme inférieure à 1 dans $R_{\infty}[1 / p]$. Soit $h \in R_{\infty}$ une fonction non nulle, d'image nulle dans $f_{\star} \mathscr{O}_{\mathfrak{X}\left(p^{\infty}\right)^{\star-m o d}}\left(\operatorname{Spf}\left(R_{\infty}\right)\right)$. Sans restreindre la généralité, on peut supposer que $|h|=1$. La surjectivité du morphisme $f$ entraîne que $h \in \mathfrak{m}_{\mathcal{O}_{\mathbb{C}_{p}}} R_{\infty}$. Une contradiction.

Corollaire 1.16. - Le faisceau $\omega_{A}^{\text {mod }}$ et les classes $H T\left(e_{1}\right), \ldots, H T\left(e_{2 g}\right)$ descendent $\grave{a} \mathfrak{X}\left(p^{\infty}\right)^{\star-\text { mod }}$.

1.17. Fibre générique. - Notons $\mathcal{X}\left(p^{\infty}\right)^{\star}$ la fibre générique de $\mathfrak{X}\left(p^{\infty}\right)^{\star-\text { mod }}$ au sens de $[\mathbf{S W}]$. Notons aussi $\mathcal{F} \mathcal{L}$ la fibre de générique de $\mathfrak{F} \mathfrak{L}$. D'après la proposition 1.15 , on a une application des périodes de Hodge-Tate $\pi_{H T}: \mathcal{X}\left(p^{\infty}\right)^{\star} \rightarrow \mathcal{F} \mathcal{L}$ et une application au niveau des espaces topologiques $\left|\pi_{H T}\right|:\left|\mathcal{X}\left(p^{\infty}\right)^{\star}\right| \rightarrow|\mathcal{F} \mathcal{L}|$. Soit $\left(X_{1}, \ldots, X_{r}\right)$ les coordonnées homogènes standard sur $\mathbb{P}^{r-1}$. Pour $1 \leq i \leq r$, on note $U_{i}$ l'ouvert rationnel standard de $\mathbb{P}^{r-1}$ défini par la conditions $X_{i} \neq 0$.

Pour tout $1 \leq i \leq r$ et $n \geq n_{0}$ soit $\mathfrak{U}_{i}\left(p^{n}\right)$ l'ouvert de $\mathfrak{X}\left(p^{n}\right)^{\star-\text { mod }}$ donné par la condition $s_{i} \neq 0$. Soit $\mathcal{U}_{i}\left(p^{n}\right)$ son tube dans $\mathcal{X}\left(p^{n}\right)^{\star}$. L'ouvert $\left|\pi_{H T}^{-1}\left(U_{i}\right)\right|$ vaut $\lim _{n \geq n_{0}}\left|\mathcal{U}_{i}\left(p^{n}\right)\right|$. Soit

$$
A_{i}\left(p^{n}\right)=\mathrm{H}^{0}\left(\mathcal{U}_{i}\left(p^{n}\right), \mathscr{O}_{\mathcal{X}\left(p^{n}\right)^{\star}}\right)
$$

et $A_{i}\left(p^{n}\right)^{0}$ la sous-algèbre des fonctions à puissance borné. Soit $A_{i}\left(p^{\infty}\right)^{0}$ la complétion $p$-adique de la limite inductive des $A_{i}\left(p^{n}\right)^{0}$ pour $n \geq n_{0}$ et $A_{i}\left(p^{\infty}\right)=A_{i}\left(p^{\infty}\right)^{0}[1 / p]$.

Théorème 1.18 ([S4], th.III.3.17). — Les assertions suivantes sont vérifiées.

1. La fibre générique $\mathcal{X}\left(p^{\infty}\right)^{\star}$ est un espace perfectoïde,

2. Les ouverts $\mathcal{U}_{i}\left(p^{n}\right)$ de $\mathcal{X}\left(p^{n}\right)^{\star}$ sont affinö̈des pour $n \geq n_{0}$,

3. On a $\pi_{H T}^{-1}\left(U_{i}\right)=\operatorname{Spa}\left(A_{i}\left(p^{\infty}\right), A_{i}\left(p^{\infty}\right)^{0}\right)$ et $\pi_{H T}^{-1}\left(U_{i}\right)$ est un espace affinoïde perfectoïde.

Démonstration. D'après [S4], th.III.3.17, (i), pour $n$ suffisamment grand, $\mathcal{U}_{i}\left(p^{n}\right)$ est affinoïde. Soit $n \geq m \geq n_{0}$. Soit $H=\operatorname{Ker}\left(G\left(\mathbb{Z} / p^{n} \mathbb{Z}\right) \rightarrow G\left(\mathbb{Z} / p^{m} \mathbb{Z}\right)\right)$. On a alors

$$
\mathscr{O}_{\mathcal{X}\left(p^{n}\right)^{\star}}^{H}=\mathscr{O}_{\mathcal{X}\left(p^{m}\right)^{\star}} \text {. }
$$

Ceci entraîne par [BGR], 6.3, prop. 3, que $A_{i}\left(p^{m}\right)=A_{i}\left(p^{n}\right)^{H}$ est une algèbre affinoide et que $\mathcal{U}_{i}\left(p^{m}\right)$ est affinoide associé à $A_{i}\left(p^{m}\right)$. Fixons un recouvrement affine $\mathfrak{X}\left(p^{n_{0}}\right)^{\star-\text { mod }}=$ $\cup_{j} \operatorname{Spf} R_{j, n_{0}}^{0}$ tel que pour tout $j$, il existe $i$ tel que $\operatorname{Spf} R_{j, n_{0}}^{0} \subset \mathfrak{U}_{i}\left(p^{n_{0}}\right)$. Soit $\operatorname{Spf} R_{j, n}^{0}$ l'image réciproque de $\operatorname{Spf} R_{j, n_{0}}^{0}$ dans $\mathfrak{X}\left(p^{n}\right)^{\star-\bmod }$ pour $n \geq n_{0}$. Soit $R_{j, \infty}^{0}$ la complétion $p$-adique de la limite inductive des $R_{j, n}^{0}$ et soit $R_{j, \infty}=R_{j, n}^{0}[1 / p]$. D'après [S4], th.III.3.17, (i), l'algèbre affinoïde $\left(A_{i}\left(p^{\infty}\right), A_{i}\left(p^{\infty}\right)^{0}\right)$ est perfectoïde. Il résulte alors de [S3], lemme 4.5, (i), que $\left(R_{j, \infty}, R_{j, \infty}^{0}\right)$ est un ouvert rationnel de $\left(A_{i}\left(p^{\infty}\right), A_{i}\left(p^{\infty}\right)^{0}\right)$, qui est donc perfectoïde. Ceci entraîne que $\mathcal{X}\left(p^{\infty}\right)^{\star}$ est un espace adique perfectoïde et que $\mathcal{X}\left(p^{\infty}\right)^{\star} \sim \lim _{n} \mathcal{X}\left(p^{n}\right)^{\star}$. L'espace $\mathcal{X}\left(p^{\infty}\right)^{\star}$ est donc bien celui construit dans [S4], sect. III.

1.19. Modification des structures entières. - On dispose pour tout $n \geq n_{0}$ des sections $s_{1}, \ldots, s_{r} \in \mathrm{H}^{0}\left(\mathfrak{X}\left(p^{n}\right)^{\star-m o d}\right.$, $\left.\operatorname{det} \omega_{A}^{\text {mod }} / p^{n-g /(p-1)}\right)$ si $p>2$ (et si $p=2$, ce sont des sections de $\left.\operatorname{det} \omega_{A}^{\bmod } / p^{n-2 g}\right)$.

Lemme 1.20. - Il existe un entier $n$ et pour tout $1 \leq i \leq r$ des section $s_{1}^{(i)}, \cdots, s_{r}^{(i)}$ dans $\mathrm{H}^{0}\left(\mathfrak{U}_{i}\left(p^{n}\right)\right.$, det $\left.\omega_{A}^{\text {mod }}\right)$ qui vérifient $s_{j}^{(i)}=s_{j} \bmod p$. 
Démonstration. D'après le théorème 1.18, $\operatorname{colim}_{n} \mathrm{H}^{0}\left(\mathcal{U}_{i}\left(p^{n}\right), \mathscr{O}_{\left.\mathcal{X}\left(p^{n}\right)^{\star}\right)}\right.$ est dense

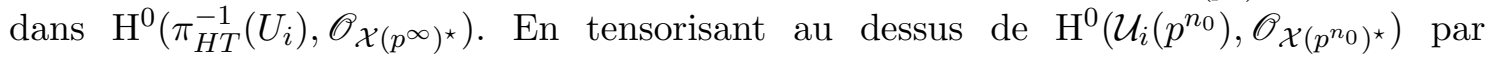
$\mathrm{H}^{0}\left(\mathcal{U}_{i}\left(p^{n_{0}}\right)\right.$, $\left.\operatorname{det} \omega_{A}\right)$, on obtient la densité de

$$
\operatorname{colim}_{n} \mathrm{H}^{0}\left(\mathcal{U}_{i}\left(p^{n}\right), \operatorname{det} \omega_{A}\right)
$$

dans $\mathrm{H}^{0}\left(\pi_{H T}^{-1}\left(U_{i}\right), \operatorname{det} \omega_{A}\right)$.

Lemme 1.21. - Il existe un entier $k$ positif et pour tout $1 \leq i \leq r$ des sections $t_{1}^{(i)}, \cdots, t_{r}^{(i)}$ dans $\mathrm{H}^{0}\left(\mathfrak{U}_{i}\left(p^{n_{0}}\right)\right.$, $\left.\operatorname{det}^{k} \omega_{A}^{\bmod }\right)$ qui vérifient $t_{j}^{(i)}=s_{j}^{k} \bmod p^{n_{0}-g /(p-1)}$ si $p>2$ (respectivement $\bmod p^{n_{0}-2 g}$ si $p=2$ ).

Démonstration. Il suffit de prendre la norme de $\mathcal{U}_{i}\left(p^{n}\right)$ à $\mathcal{U}_{i}\left(p^{n_{0}}\right) \operatorname{des} \operatorname{sections} s_{j}^{(i)}$.

Théorème 1.22 ([S4], p.72). — Pour tout $n \geq n_{0}$, il existe un modèle formel $\mathfrak{X}\left(p^{n}\right)^{\star-H T}$ de $\mathcal{X}\left(p^{n}\right)^{\star}$ qui est recouvert par les schémas formels affines

$$
\mathfrak{V}_{i}\left(p^{n}\right)=\operatorname{Spf} \mathrm{H}^{0}\left(\mathfrak{U}_{i}\left(p^{n}\right), \mathscr{O}_{\mathfrak{X}\left(p^{n}\right)^{\star-m o d}}\right) .
$$

Il s'insère dans un diagramme

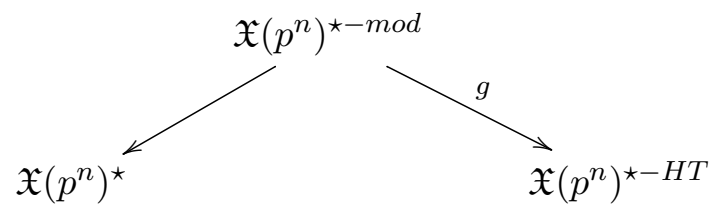

Le faisceau inversible $\operatorname{det}^{k} \omega_{A}^{\text {mod }}$ défini sur $\mathfrak{X}\left(p^{n}\right)^{\star-\text { mod }}$ descend en un faisceau inver-

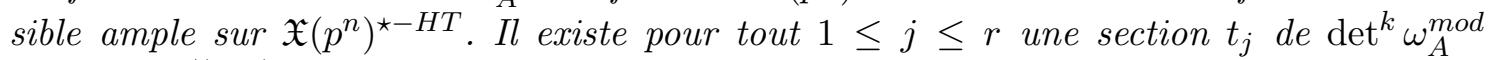
$\bmod p^{n_{0}-g /(p-1)}$ si $p>2$ (respectivement $\bmod p^{n_{0}-2 g}$ si $p=2$ ) qui recolle les sections $t_{j}^{(1)}, \cdots, t_{j}^{(r)}$. Pour tout $n \geq m \geq n_{0}$, on a un morphisme fini

$$
\mathfrak{X}\left(p^{n}\right)^{\star-H T} \longrightarrow \mathfrak{X}\left(p^{m}\right)^{\star-H T}
$$

et le diagramme 1.22.A est fonctoriel en $n$ et en le niveau $K^{p}$.

Remarque 1.23. - Pour $n$ suffisamment grand mais non explicite, le faisceau $\operatorname{det}\left(\omega_{A}^{\text {mod }}\right)$ descend à $\mathfrak{X}\left(p^{n}\right)^{\star-H T}$.

Remarque 1.24. - La démonstration du théorème précédent utilise de façon essentielle [S4, th.III.3.17]. Existe-t-il une démonstration qui n'utilise que des niveaux finis?

Remarque 1.25. - On a montré que $\omega_{A}^{\text {mod }}$ est semi-ample sur $\mathfrak{X}\left(p^{n}\right)^{*-\text { mod }}$ et que $\mathfrak{X}\left(p^{n}\right)^{*-H T}$ est sa factorisation de Stein. De plus, les sections $s_{1}, \cdots, s_{r}$ sont sans point base sur

$$
\mathfrak{X}\left(p^{n}\right)^{*-\bmod } \times \operatorname{Spec}\left(\mathcal{O}_{\mathbb{C}_{p}} / p^{n-g /(p-1)}\right)
$$

si $p>2$ (respectivement $\bmod p^{n-2 g}$ si $p=2$ ) et les sections $t_{1}, \cdots, t_{r}$ sont sans point base sur

$$
\mathfrak{X}\left(p^{n}\right)^{*-H T} \times \operatorname{Spec}\left(\mathcal{O}_{\mathbb{C}_{p}} / p^{n_{0}-g /(p-1)}\right)
$$

si $p>2\left(\right.$ respectivement $\bmod p^{n_{0}-2 g}$ si $\left.p=2\right)$.

Remarque 1.26. - Le théorème 1.22 permet éventuellement de donner des équations de $\mathfrak{X}\left(p^{n}\right)^{*-H T}$ dans certains cas explicites, comme celui de la courbe de Legendre. On déduit aussi de ce théorème que la contraction $\mathfrak{X}\left(p^{n}\right)^{*-\bmod } \rightarrow \mathfrak{X}\left(p^{n}\right)^{*-H T}$ identifie des points ordinaires et des points non ordinaires, et des points de l'intérieur avec des points du bord. C'est pourquoi on ne dispose sur $\mathfrak{X}\left(p^{n}\right)^{*-H T}$ ni de schéma semi-abélien universel, 
ni de notion de bord, ni de notion de lieu ordinaire ni d'aucune stratification comme celles de Newton ou Ekedhal-Oort (voir la note de bas de page p.72 de [S4]).

Remarque 1.27. - On peut considérer la limite inverse $\mathfrak{X}\left(p^{\infty}\right)^{\star-H T}$ des schémas formels $\mathfrak{X}\left(p^{n}\right)^{\star-H T}$. On dispose alors du diagramme suivant :

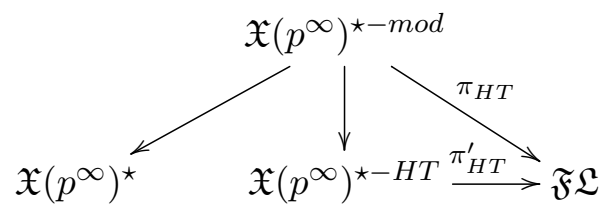

où $\pi_{H T}^{\prime *} \mathscr{O}_{\mathfrak{F} \mathfrak{L}}(1)=\operatorname{det}\left(\omega_{A}^{\text {mod }}\right)$. Ajoutons un indice $a$ pour indiquer qu'on regarde le schéma sur $\operatorname{Spec}\left(\mathcal{O}_{\mathbb{C}_{p}} / p^{a}\right)$ obtenu par réduction modulo $p^{a}$ d'un schéma formel sur $\operatorname{Spf}\left(\mathcal{O}_{\mathbb{C}_{p}}\right)$. Par exemple, $\mathfrak{X}_{a}$ est la variété de Siegel $\operatorname{sur} \operatorname{Spec}\left(\mathcal{O}_{\mathbb{C}_{p}} / p^{a}\right)$. Alors pour tout $a$, le morphisme

$$
\mathfrak{X}\left(p^{\infty}\right)_{a}^{\star-H T} \rightarrow \mathfrak{F} \mathfrak{L}_{a}
$$

se factorise par $\mathfrak{X}\left(p^{\infty}\right)_{a}^{\star-H T} \rightarrow \mathfrak{X}\left(p^{n}\right)_{a}^{\star-H T} \rightarrow \mathfrak{F} \mathfrak{L}_{a}$ pour $n$ suffisamment grand dépendant de $a$ (utiliser une variante du lemme 1.20). Le morphisme de schémas de type fini $\mathfrak{X}\left(p^{n}\right)_{a}^{\star-H T} \rightarrow \mathfrak{F} \mathfrak{L}_{a}$ est propre et affine, il est donc fini. Ceci montre que le morphisme $\pi_{H T}^{\prime}$ est pro-fini.

\section{Le cas Hodge}

Dans cette section nous expliquons comment on étend les résultats précédents aux variétés de Shimura de type Hodge dans le contexte de [S4, ch.V].

2.1. Variétés de Shimura de type Hodge. - Soit $G$ un groupe réductif sur $\mathbb{Q}$ de groupe dérivé simplement connexe muni d'une classe de $G(\mathbb{R})$-conjugaison $\mathfrak{D}$ de morphismes $u: U(1) \rightarrow G_{\mathbb{R}}^{a d}$ tels que ad $u(-1)$ soit une involution de Cartan et $\mu=u_{\mathbb{C}}$ : $\mathbb{G}_{m, \mathbb{C}} \rightarrow G_{\mathbb{C}}$ soit minuscule. Soit $K_{G} \subset G\left(\mathbb{A}_{f}\right)$ un sous-groupe compact ouvert des points de $G$ à valeurs dans les adèles finies de $\mathbb{Q}$. Supposons $K_{G}=K_{G}^{p} \cdot K_{G, p}$ avec $K_{G}^{p} \subset G\left(\mathbb{A}_{f}^{p}\right)$ et $K_{G, p} \subset G\left(\mathbb{Q}_{p}\right)$. Notons

$$
X_{G}\left(K_{G}\right)=G(\mathbb{Q}) \backslash\left(\mathfrak{D} \times G\left(\mathbb{A}_{f}\right) / K_{G}\right) .
$$

D'après un résultat classique de Baily-Borel, c'est une variété algébrique complexe. Choisissons un plongement $\mathbb{C} \hookrightarrow \mathbb{C}_{p}$ et notons toujours $X_{G}\left(K_{G}\right)$ le changement de base à $\mathbb{C}_{p}$. Nous ferons de même pour toute variété complexe sans l'indiquer dans les notations.

Nous supposerons désormais que $(G, \mathfrak{D})$ est de type Hodge, ce qui signifie qu'il existe un entier $g$ et un plongement $(G, \mathfrak{D}) \hookrightarrow\left(\mathrm{GSp}_{2 g}, \mathfrak{H}^{ \pm}\right)$où $\mathfrak{H}^{ \pm}$désigne l'espace symétrique de Siegel de genre $g$. Soit $n$ un entier. Supposons que

$$
K_{G, p}=G\left(\mathbb{Q}_{p}\right) \cap \operatorname{Ker}\left(\mathrm{GSp}_{2 g}\left(\mathbb{Z}_{p}\right) \rightarrow \operatorname{GSp}_{2 g}\left(\mathbb{Z} / p^{n}\right)\right) .
$$

Cette condition est satisfaite pour un ensemble cofinal de $K_{G, p}$. Nous noterons alors $X_{G}\left(p^{n}, K_{G}^{p}\right)=X_{G}\left(K_{G}\right)$. Si le contexte est clair, on supprime même $K_{G}^{p}$ de la notation et on pose $X_{G}\left(p^{n}\right)=X_{G}\left(p^{n}, K_{G}^{p}\right)$. Notons $\mathfrak{L}$ l'ensemble ordonné par inclusion des sous-groupes compacts ouverts $K^{p} \subset \mathrm{GSp}_{2 g}\left(\mathbb{A}_{f}^{p}\right)$ tels que $K_{G}^{p}=G\left(\mathbb{A}_{f}^{p}\right) \cap K^{p}$, que $K_{p} \cdot K^{p}$ soit net et que le morphisme $(G, \mathfrak{D}) \hookrightarrow\left(\mathrm{GSp}_{2 g}, \mathfrak{H}^{ \pm}\right)$induise une immersion fermée

$$
X_{G}\left(p^{n}\right) \hookrightarrow X\left(p^{n}, K^{p}\right)
$$

sur $\operatorname{Spec}\left(\mathbb{C}_{p}\right)$. Ici $X\left(p^{n}, K^{p}\right)$ désigne la variété de Siegel de genre $g$, de niveau $K^{p}$ hors $p$ et de niveau plein $p^{n}$ en $p$ sur $\operatorname{Spec}\left(\mathbb{C}_{p}\right)$. D'après $[\mathbf{K i}$, lem.2.1.2], l'ensemble $\mathfrak{L}$ est non vide 
si $n \geq n_{0}$. De plus, d'après la démonstration de [Ki, lem.2.1.2] (voir aussi le début de la démonstration de $\left[\mathbf{D}\right.$, prop.1.15]), si $K^{p} \in \mathfrak{L}$ et $K^{\prime p} \subset K^{p}$ vérifie $K_{G}^{p}=G\left(\mathbb{A}_{f}^{p}\right) \cap K^{\prime p}$, alors $K^{\prime p} \in \mathfrak{L}$.

D'après $[\mathbf{H 2}]$, la donnée de $\mathcal{S}$ détermine une compactification toroïdale $X_{G}\left(p^{n}\right)^{\text {tor }}$ sur $\mathbb{C}_{p}$ ainsi qu'un morphisme

$$
X_{G}\left(p^{n}\right)^{\text {tor }} \rightarrow X\left(p^{n}, K^{p}\right)^{\text {tor }}
$$

pour tout $K^{p}$ dans $\mathfrak{L}$. Par construction (voir [H2, lem.3.1]), ce morphisme est fini et identifie $X_{G}\left(p^{n}\right)^{t o r}$ à la normalisée de l'adhérence schématique de son image dans $X\left(p^{n}, K^{p}\right)^{t o r}$. Notons $\mathcal{X}_{G}\left(p^{n}\right)^{\text {tor }}$ la variété rigide associée sur $\mathbb{C}_{p}$. On dispose également de la compactification minimale $X_{G}\left(p^{n}\right)^{*}$ qu'on voit comme un schéma sur $\mathbb{C}_{p}$ et d'un morphisme fini

$$
X_{G}\left(p^{n}\right)^{*} \rightarrow X\left(p^{n}, K^{p}\right)^{*}
$$

qui identifie $X_{G}\left(p^{n}\right)^{*}$ à la normalisée de l'adhérence de son image dans $X\left(p^{n}, K^{p}\right)^{*}$ pour tout $K^{p} \in \mathfrak{L}$. On note aussi $\mathcal{X}_{G}\left(p^{n}\right)^{*}$ la variété rigide associée. On a un diagramme commutatif pour tout $K^{p} \in \mathfrak{L}$.

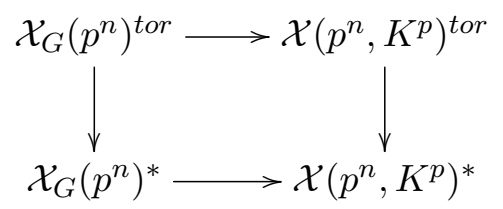

2.2. Modèles entiers des compactifications. - Supposons $n \geq n_{0}$ où $n_{0}$ dépend de $p$ et de $g$ donc du plongement symplectique, et a été décrit dans la partie 1 .

2.2.1. Modèles des compactifications toroidales. - Notons $\mathfrak{X}_{G}\left(p^{n}\right)^{\text {tor-mod }}$ la normalisée de l'adhérence schématique de $\mathcal{X}_{G}\left(p^{n}\right)^{\text {tor }}$ dans $\mathfrak{X}\left(p^{n}, K^{p}\right)^{\text {tor-mod }}$ pour tout $K^{p} \in \mathfrak{L}$. C'est un schéma formel qui dépend a priori du choix du plongement $(G, \mathfrak{D}) \hookrightarrow\left(\mathrm{GSp}_{2 g}, \mathfrak{H}^{ \pm}\right)$.

Lemme 2.3. - Le schéma formel $\mathfrak{X}_{G}\left(p^{n}\right)^{\text {tor-mod }}$ est indépendant du choix de $K^{p} \in \mathfrak{L}$.

Démonstration. Si $K^{\prime p} \subset K^{p}$ sont deux éléments de $\mathfrak{L}$, notons $\mathfrak{X}_{G}\left(p^{n}, K^{p}\right)^{\text {tor-mod }}$ et $\mathfrak{X}_{G}\left(p^{n}, K^{\prime p}\right)^{\text {tor-mod }}$ les deux schémas formels associés. On obtient un morphisme

$$
\mathfrak{X}_{G}\left(p^{n}, K^{\prime p}\right)^{\text {tor-mod }} \longrightarrow \mathfrak{X}_{G}\left(p^{n}, K^{p}\right)^{\text {tor-mod }}
$$

qui est fini birationnel entre schémas formels normaux, donc un isomorphisme. Si $K^{\prime p}$ et $K^{p}$ sont quelconques dans $\mathfrak{L}$, on en déduit le même résultat en considérant $K^{p} \cap K^{\prime p}$ qui reste bien dans $\mathfrak{L}$.

Réindiquons juste pour une seconde $\mathfrak{X}_{G}\left(p^{n}, K_{G}^{p}, \mathcal{S}\right)^{\text {tor-mod }}=\mathfrak{X}_{G}\left(p^{n}\right)^{\text {tor-mod }}$ pour marquer la dépendance en $K_{G}^{p}$ et $\mathcal{S}$. Lorsque $K_{G}^{\prime p} \subset K_{G}^{p}$ on dispose d'un morphisme fini

$$
\mathfrak{X}_{G}\left(p^{n}, K_{G}^{\prime p}, \mathcal{S}\right)^{\text {tor-mod }} \longrightarrow \mathfrak{X}_{G}\left(p^{n}, K_{G}^{p}, \mathcal{S}\right)^{\text {tor-mod }}
$$

et lorsque $\mathcal{S}^{\prime}$ raffine $\mathcal{S}$ on dispose d'un morphisme propre

$$
\mathfrak{X}_{G}\left(p^{n}, K_{G}^{p}, \mathcal{S}^{\prime}\right)^{\text {tor-mod }} \longrightarrow \mathfrak{X}_{G}\left(p^{n}, K_{G}^{p}, \mathcal{S}\right)^{\text {tor-mod }}
$$

et l'on obtient donc une double tour

$$
\left(\mathfrak{X}_{G}\left(p^{n}, K_{G}^{p}, \mathcal{S}\right)^{\text {tor-mod }}\right)_{K_{G}^{p}, \mathcal{S}}
$$

munie d'une action canonique de $G\left(\mathbb{A}_{f}^{p}\right)$ étendant l'action usuelle en fibre générique. Plus précisémment, pour tous sous-groupes compacts ouverts $K_{G}^{\prime p} \subset K_{G}^{p} \subset G\left(\mathbb{A}_{f}^{p}\right)$, tout $g \in$ 
$G\left(\mathbb{A}_{f}^{p}\right)$ tel que $g^{-1} \cdot K_{G}^{\prime p} \cdot g \subset K_{G}^{p}$ et toute décomposition polyhédrale $\mathcal{S}$ comme dans A.1, il existe une décomposition polyhédrale $\mathcal{S}^{\prime}$ comme dans A.1 et un morphisme propre surjectif

$$
[g]: \mathfrak{X}_{G}\left(p^{n}, K_{G}^{\prime p}, \mathcal{S}^{\prime}\right)^{\text {tor-mod }} \longrightarrow \mathfrak{X}_{G}\left(p^{n}, K_{G}^{p}, \mathcal{S}\right)^{\text {tor-mod }}
$$

qui redonne le morphisme de Hecke usuel sur le complémentaire du bord de la fibre générique. Lorsque $n \geq m$ on obtient de plus un morphisme fini

$$
\mathfrak{X}_{G}\left(p^{n}, K_{G}^{p}, \mathcal{S}\right)^{\text {tor-mod }} \rightarrow \mathfrak{X}_{G}\left(p^{m}, K_{G}^{p}, \mathcal{S}\right)^{\text {tor-mod }}
$$

et une triple tour

$$
\left(\mathfrak{X}_{G}\left(p^{n}, K_{G}^{p}, \mathcal{S}\right)^{\text {tor-mod }}\right)_{K_{G}^{g}, \mathcal{S}, n \geq n_{0}}
$$

munie d'une action de $G\left(\mathbb{A}_{f}^{p}\right)$.

2.3.1. Modèles de la compactification minimale. - Notons $\mathfrak{X}_{G}\left(p^{n}\right)^{*-\bmod }$ la normalisée de l'adhérence schématique de $\mathcal{X}_{G}\left(p^{n}\right)^{*}$ dans $\mathfrak{X}\left(p^{n}, K^{p}\right)^{*-\text { mod }}$ pour tout $K^{p} \in \mathfrak{L}$. Elle ne dépend pas du choix de $K^{p} \in \mathfrak{L}$. Les schémas formels $\mathfrak{X}_{G}\left(p^{n}\right)^{*-\text { mod }}$ s'agencent en une tour stable par $G\left(\mathbb{A}_{f}^{p}\right)$ lorsque $K_{G}^{p}$ varie. Notons de même $\mathfrak{X}_{G}\left(p^{n}\right)^{*-H T}$ la normalisée de l'adhérence schématique de $\mathcal{X}_{G}\left(p^{n}\right)^{*}$ dans $\mathfrak{X}\left(p^{n}, K^{p}\right)^{*-H T}$ qui s'agencent encore en une tour stable par $G\left(\mathbb{A}_{f}^{p}\right)$. Le morphisme naturel $\pi: \mathfrak{X}_{G}\left(p^{n}\right)^{*-\bmod } \rightarrow \mathfrak{X}_{G}\left(p^{n}\right)^{*-H T}$ vérifie $\pi_{*}(\mathscr{O})=\mathscr{O}$ par normalité. On obtient donc pour tout $K^{p} \in \mathfrak{L}$ un diagramme commutatif à flèches horizontales finies et à flèches verticales propres birationnelles surjectives

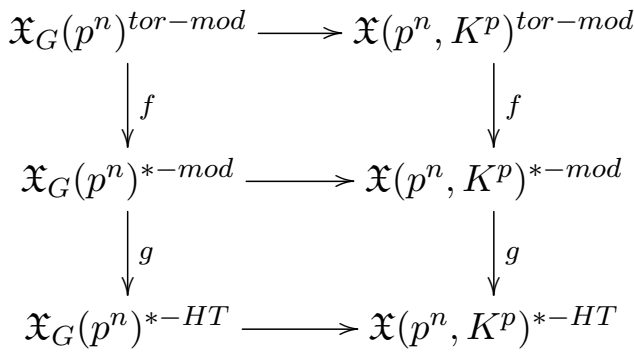

On obtient donc par image inverse un schéma semi-abélien $A$ sur $\mathfrak{X}_{G}\left(p^{n}\right)^{\text {tor-mod }}$, un fibré inversible $\operatorname{det} \omega_{A}^{\text {mod }}$ sur $\mathfrak{X}_{G}\left(p^{n}\right)^{*-m o d}$ et des sections $s_{1}, \cdots, s_{r} \operatorname{de} \operatorname{det} \omega_{A} / p^{n-g /(p-1)}$ si $p>2$ (respectivement $\operatorname{det} \omega_{A} / p^{n-2 g}$ si $p=2$ ). De plus, il existe un entier $k$ (le même que dans la partie 1) tel que $\operatorname{det}^{k} \omega_{A}^{\text {mod }}$ descende en un fibré inversible ample sur $\mathfrak{X}_{G}\left(p^{n}\right)^{*-H T}$ et que les sections $t_{1}=s_{1}^{k}, \cdots, t_{r}=s_{r}^{k}$ descendent en des sections de $\operatorname{det}^{k} \omega_{A}^{\text {mod }}$ modulo une puissance de $p$ convenable sur $\mathfrak{X}_{G}\left(p^{n}\right)^{*-H T}$.

Remarque 2.4. - Le schéma $A$ n'est pas canonique car même sa dimension dépend du plongement $(G, \mathfrak{D}) \hookrightarrow\left(\mathrm{GSp}_{2 g}, \mathfrak{H}^{ \pm}\right)$. Cela n'a aucune importance pour l'application que nous avons en vue dans la partie 3 .

Notons $\mathfrak{U}_{G, i}\left(p^{n}\right)$ l'ouvert formel de $\mathfrak{X}_{G}\left(p^{n}\right)^{*-\text { mod }}$ où $s_{i}$ est non nul. Le schéma $\mathfrak{X}_{G}\left(p^{n}\right)^{*-\bmod }$ est alors recouvert par les $\mathfrak{U}_{G, i}\left(p^{n}\right)$ pour $1 \leq i \leq r$ puisque c'est le cas de $\mathfrak{X}\left(p^{n}\right)^{*-\text { mod }}$. On a donc montré la proposition suivante.

Proposition 2.5 ([S4], p.72). - Pour tout $n \geq n_{0}$, il existe un modèle formel $\mathfrak{X}_{G}\left(p^{n}\right)^{*-H T}$ qui est recouvert par les schémas formels affines

$$
\mathfrak{V}_{G, i}\left(p^{n}\right)=\operatorname{Spf} \mathrm{H}^{0}\left(\mathfrak{U}_{G, i}\left(p^{n}\right), \mathscr{O}_{\mathfrak{X}_{G}\left(p^{n}\right)^{*-m o d}}\right) .
$$

Il existe un morphisme canonique $g: \mathfrak{X}_{G}\left(p^{n}\right)^{*-\bmod } \rightarrow \mathfrak{X}_{G}\left(p^{n}\right)^{*-H T}$. Pour tout $n \geq m \geq n_{0}$ il existe un morphisme fini $\mathfrak{X}_{G}\left(p^{n}\right)^{*-H T} \rightarrow \mathfrak{X}_{G}\left(p^{m}\right)^{*-H T}$ et le morphisme $g$ est fonctoriel en $n$ et en le niveau auxiliaire $K_{G}^{p}$. Le faisceau inversible $\operatorname{det}^{k} \omega_{A}^{\text {mod }}$ est ample 
sur $\mathfrak{X}_{G}\left(p^{n}\right)^{*-H T}$ et est muni d'une famille de sections $t_{1}, \cdots, t_{r}$ modulo $p^{n_{0}-g /(p-1)}$ si $p>2$ (respectivement modulo $p^{n_{0}-2 g}$ si $p=2$ ) qui sont sans point base.

Remarque 2.6. - Le contexte dans lequel nous nous sommes placés nous autorise à travailler avec les variétés de Shimura connexes, comme par exemple celle associée au groupe $G=\mathrm{Sp}_{2 g}$ sur $\mathbb{Q}$.

2.7. Modèles des faisceaux automorphes. - Fixons $u \in \mathfrak{D}$ et notons $\mu=u_{\mathbb{C}}$ : $\mathbb{G}_{m, \mathbb{C}} \rightarrow G_{\mathbb{C}}$ que l'on transfère à $\mathbb{C}_{p}$. Notons $G_{\mathbb{C}}^{\text {der }}$ le sous-groupe dérivé de $G_{\mathbb{C}}$, notons $P_{G}$ le sous-groupe parabolique de $G_{\mathbb{C}_{p}}^{\text {der }}$ qui stabilise $\mu$ et $M_{G}$ son quotient de Lévi. Pour toute représentation algébrique $\kappa$ de $M_{G}$ sur un $\mathbb{C}_{p}$-espace vectoriel $V_{\kappa}$ et tout $n \geq 0$, on dispose du faisceau automorphe cohérent $\omega^{\kappa}$ sur $X_{G}\left(p^{n}\right)^{t o r}$ [Mi, III.2,V.6]. Il est localement libre et ses fibres sont isomorphes à $V_{\kappa}$. Notre but est de définir un modèle entier de ce faisceau qui soit cohérent et stable par les opérateurs de Hecke sur $\mathfrak{X}_{G}\left(p^{n}\right)^{\text {tor-mod }}$ lorsque $n \geq n_{0}$.

Proposition 2.8. - Pour toute représentation algébrique $\kappa$ de $M_{G}$, le faisceau automorphe cohérent $\omega^{\kappa}$ sur $X_{G}\left(p^{n}\right)^{\text {tor-mod }}$ peut s'étendre en un faisceau cohérent $\omega^{\kappa}$ sur $\mathfrak{X}_{G}\left(p^{n}\right)^{\text {tor-mod }}$ pour tout $n \geq n_{0}$ muni d'une action de $G\left(\mathbb{A}_{f}^{p}\right)$ sur la double tour

$$
\left(\mathfrak{X}_{G}\left(p^{n}, K_{G}^{p}, \mathcal{S}\right)^{\text {tor-mod }}\right)_{K_{G}^{p}, \mathcal{S}}
$$

et de manière compatible au morphisme $\mathfrak{X}_{G}\left(p^{n}\right)^{\text {tor-mod }} \rightarrow \mathfrak{X}_{G}\left(p^{m}\right)^{\text {tor-mod }}$ pour tout $n \geq$ $m \geq n_{0}$.

Démonstration. Notons $\mu_{\mathrm{GSp}}: \mathbb{G}_{m, \mathbb{C}_{p}} \rightarrow \mathrm{GSp}_{2 g, \mathbb{C}_{p}}$ le cocaractère minuscule canoniquement associé à la variété de Siegel, $P_{\mathrm{Sp}}$ son stabilisateur dans $\mathrm{Sp}_{2 g, \mathbb{C}_{p}}$ et $M_{\mathrm{Sp}}$ le quotient de Lévi de $P_{\mathrm{Sp}}$. On a un isomorphisme canonique $M_{\mathrm{Sp}} \simeq \mathrm{GL}_{g}$ et $M_{\mathrm{Sp}}$ agit fidèlement sur St $=\mathbb{C}_{p}^{g}$. Le plongement $(G, \mathfrak{D}) \hookrightarrow\left(\mathrm{GSp}_{2 g}, \mathfrak{H}^{ \pm}\right)$définit une injection $M_{G} \hookrightarrow M_{\mathrm{Sp}}$ et une représentation fidèle de $M_{G}$ sur St. D'après [DM, prop.2.20], il existe des entiers $r$ et $s$ et une surjection $M_{G}$-équivariante $\mathrm{St}^{r} \otimes\left(\mathrm{St}^{\vee}\right)^{s} \rightarrow V_{\kappa}$ où $\mathrm{St}^{\vee}$ désigne la représentation duale. Le plongement $(G, \mathfrak{D}) \hookrightarrow\left(\mathrm{GSp}_{2 g}, \mathfrak{H}^{ \pm}\right)$définit également un schéma semi-abélien $A$ et son faisceau localement libre $\omega_{A}$ des 1-formes différentielles invariantes sur $\mathfrak{X}_{G}\left(p^{n}\right)^{\text {tor-mod }}$. Ce faisceau est bien muni d'action de $G\left(\mathbb{A}_{f}^{p}\right)$ sur la double tour et est compatible au morphisme $\mathfrak{X}_{G}\left(p^{n}\right)^{\text {tor-mod }} \rightarrow \mathfrak{X}_{G}\left(p^{m}\right)^{\text {tor-mod }}$ pour $n \geq m \geq n_{0}$. De plus, la restriction de $\omega_{A}$ à $X_{G}\left(p^{n}\right)$ coïncide par construction avec le faisceau automorphe associé à la représentation St de $M_{G}$. On obtient donc une surjection $\left.\omega_{A}^{r} \otimes\left(\omega_{A}^{\vee}\right)^{s}\right|_{X_{G}\left(p^{n}\right)} \rightarrow \omega^{\kappa}$ de faisceaux sur $X_{G}\left(p^{n}\right)$ et on peut prolonger $\omega^{\kappa}$ sur $\mathfrak{X}_{G}\left(p^{n}\right)$ en considérant l'image de la structure entière $\omega_{A}^{r} \otimes\left(\omega_{A}^{\vee}\right)^{s}$. L'équivariance sous $G\left(\mathbb{A}_{f}^{p}\right)$ est automatique.

Remarque 2.9. - Supposons le groupe $G$ non ramifié sur $\mathbb{Q}_{p}$ et que dans le cas Hodge non PEL de type (A) ou (C) on a $p>2$ (voir [Ma, rem.4.6.5] pour ce qu'il est possible de faire lorsque $p=2$ ). Le faisceau $\omega^{\kappa}$ admet alors un prolongement canonique localement libre sur $\mathfrak{X}_{G}\left(p^{n}\right)^{\text {tor-mod }}$ stable par les opérateurs de Hecke. En effet, un modèle entier $\mathfrak{X}_{G}(1)^{\text {tor }}$ de la compactification toroïdale $X_{G}(1)^{\text {tor }}$ a été construit en niveau hyperspécial dans $[\mathbf{L 1}]$ et $[\mathbf{M a}]$. Le faisceau $\omega^{\kappa}$ se prolonge canoniquement de manière localement libre sur $\mathfrak{X}_{G}(1)^{\text {tor }}$ (voir [Ki, cor.2.3.9] et [Ma, par.4.4.4]) et il résulte de notre construction qu'on dispose d'un morphisme $\mathfrak{X}_{G}\left(p^{n}\right)^{\text {tor-mod }} \rightarrow \mathfrak{X}_{G}(1)^{\text {tor }}$. Il suffit donc de tirer ce prolongement localement libre.

Par contre, le cas d'une variété de Hilbert associée à un corps totalement réel dans lequel $p$ est ramifié montre qu'il n'est pas vrai que $\omega^{\kappa}$ se prolonge toujours de manière localement libre au modèle entier de Kottwitz, même avant compactification. 
Notons $D_{\eta}$ le diviseur de bord de $X_{G}\left(p^{n}\right)^{\text {tor }}$ et $D$ son adhérence schématique dans $\mathfrak{X}_{G}\left(p^{n}\right)^{\text {tor-mod }}$, qui est un diviseur de Cartier. On peut donc définir un modèle entier $\omega^{\kappa}(-D)$ sur $\mathfrak{X}_{G}\left(p^{n}\right)^{\text {tor-mod }}$ du faisceau automorphe cuspidal $\omega^{\kappa}\left(-D_{\eta}\right)$ sur $X_{G}\left(p^{n}\right)^{\text {tor }}$. Il est également muni d'une action de $G\left(\mathbb{A}_{f}^{p}\right)$ lorsqu'on l'évalue sur la double tour.

\section{Application}

On va à présent utiliser les résultats géométriques des sections précédentes pour associer des représentations galoisiennes à des classes de cohomologie cohérente de caractéristique nulle. On se place dans le cadre de la partie 2.

3.1. Formes modulaires. - Soit $\kappa$ une représentation algébrique irréductible de $M_{G}$ sur un $\mathbb{C}_{p}$-espace vectoriel de dimension finie. On dispose par la proposition $2.8 \mathrm{du}$ faisceau $\omega^{\kappa}$ sur $\mathfrak{X}_{G}\left(p^{n}\right)^{\text {tor-mod }}$ pour $n \geq n_{0}$ ainsi que de sa variante cuspidale $\omega^{\kappa}(-D)$. On notera 1 la représentation obtenue par composition

$$
M_{G} \longrightarrow \mathrm{GL}_{g, \mathbb{C}_{p}} \stackrel{\text { det }}{\longrightarrow} \mathbb{G}_{m, \mathbb{C}_{p}}
$$

dépendant du plongement $G \hookrightarrow \mathrm{GSp}_{2 g}$ et on obtient en conséquence les fibrés inversibles $\omega \underline{1}$ et $\omega^{1}(-D)$. On dispose aussi d'un faisceau $\operatorname{det} \omega_{A}^{\bmod }$ sur $\mathfrak{X}_{G}\left(p^{n}\right)^{\star-\bmod }$ et il existe un entier positif $k$ tel que $\operatorname{det}^{k} \omega_{A}^{\text {mod }}$ descende en un faisceau inversible ample sur $\mathfrak{X}_{G}\left(p^{n}\right)^{\star-H T}$. Par ailleurs, l'image inverse de $\operatorname{det} \omega_{A}^{\text {mod }}$ sur $\mathfrak{X}_{G}\left(p^{n}\right)^{\text {tor-mod }}$ est contenue dans le faisceau $\omega^{1}=\operatorname{det} \omega_{A}$ et ces deux faisceaux sont égaux en fibre générique.

On note $M_{\kappa}\left(p^{n}\right)=\mathrm{H}^{0}\left(\mathcal{X}_{G}\left(p^{n}\right)^{\text {tor }}, \omega^{\kappa}\right)$ le $\mathbb{C}_{p^{-}}$-espace des formes modulaires holomorphes pour le groupe $G$ de poids $\kappa$ et niveau $K^{p}$ hors de $p$ et $p^{n}$ en $p$, et on note $S_{\kappa}\left(p^{n}\right)=$ $\mathrm{H}^{0}\left(\mathcal{X}_{G}\left(p^{n}\right)^{t o r}, \omega^{\kappa}(-D)\right)$ le sous-module des formes cuspidales. Soit $\mathbb{T}$ l'algèbre de Hecke abstraite non ramifiée à coefficient dans $\mathbb{Z}_{p}$ pour $G$ engendrée par les opérateurs de niveau premier à $p$ et $K^{p}$. L'algèbre $\mathbb{T}$ agit sur $S_{\kappa}\left(p^{n}\right)$.

3.2. Le résultat. - Fixons une représentation irréductible $\kappa$ et $i$ un entier positif. Soit

$$
S_{\kappa}^{i}\left(p^{n}\right)=\mathrm{H}^{i}\left(\mathcal{X}_{G}\left(p^{n}\right)^{t o r}, \omega^{\kappa}(-D)\right) \text { et } M_{\kappa}^{i}\left(p^{n}\right)=\mathrm{H}^{i}\left(\mathcal{X}_{G}\left(p^{n}\right)^{t o r}, \omega^{\kappa}\right) \text {. }
$$

Lemme 3.3. - Les espaces $S_{\kappa}^{i}\left(p^{n}\right)$ et $M_{\kappa}^{i}\left(p^{n}\right)$ ne dépendent pas du choix combinatoire intervenant dans la définition de la compactification torö̈dale.

Démonstration. Soit $\mathcal{S}^{\prime}$ un choix combinatoire qui raffine $\mathcal{S}$. Remarquons ce choix dans notre notation des compactifications. On a alors un morphisme

$$
\alpha: \mathcal{X}_{G}\left(p^{n}, \mathcal{S}^{\prime}\right)^{\text {tor }} \rightarrow \mathcal{X}\left(p^{n}, \mathcal{S}\right)^{\text {tor }}
$$

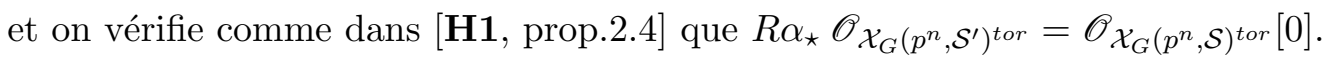

Rappelons l'existence d'un morphisme composé $h=g \circ f$ avec

$$
\mathfrak{X}_{G}\left(p^{n}\right)^{\text {tor-mod }} \stackrel{f}{\longrightarrow} \mathfrak{X}_{G}\left(p^{n}\right)^{*-\bmod } \stackrel{g}{\longrightarrow} \mathfrak{X}_{G}\left(p^{n}\right)^{*-H T} .
$$

Notons alors $\mathbb{S}_{\kappa}^{i}\left(p^{n}\right)=\mathrm{H}^{i}\left(\mathfrak{X}_{G}\left(p^{n}\right)^{\star-H T}, h_{\star} \omega^{\kappa}(-D)\right)$.

Remarque 3.4. - Nous allons utiliser l'analogue des résultats de [LSt] dans le cas Hodge sur $\mathbb{C}_{p}$, l'article $[\mathbf{L S t}]$ se plaçant dans le cas PEL. La même démonstration reste valable dans le cas Hodge si l'on dispose de l'analogue des théorèmes d'annulation de cohomologie cohérente complexe de [LSu] (les énoncés d'annulation en cohomologie de Betti sont vrais pour toute variété de Shimura par [LSw, cor.5.6]). Remarquons que lorsque $\kappa$ est une puissance tensorielle de 1, la formule de projection et [LSt, par.4] montrent directement le résultat voulu dans le cas Hodge. 
Proposition 3.5. - Les points suivants sont vérifiés.

1. On a un isomorphisme canonique $\mathbb{S}_{\kappa}^{i}\left(p^{n}\right) \otimes_{\mathcal{O}_{\mathbb{C}_{p}}} \mathbb{C}_{p}=S_{\kappa}^{i}\left(p^{n}\right)$.

2. Le module $\mathbb{S}_{\kappa}^{i}\left(p^{n}\right)$ est indépendant du choix combinatoire intervenant dans la définition du morphisme $f$,

3. On a une action de l'algèbre de Hecke $\mathbb{T}$ sur $\mathbb{S}_{\kappa}^{i}\left(p^{n}\right)$.

Démonstration. D'après le résultat principal de [LSt], on a $R^{i} f_{\star} \omega^{\kappa}[1 / p]=0$ pour $i>0$ et donc $\mathrm{H}^{i}\left(\mathcal{X}_{G}\left(p^{n}\right)^{t o r}, \omega^{\kappa}(-D)\right)=\mathrm{H}^{i}\left(\mathcal{X}_{G}\left(p^{n}\right)^{\star}, f_{\star} \omega^{\kappa}(-D)\right)$. Pour le second point, soit $\mathcal{S}^{\prime}$ un choix combinatoire qui raffine $\mathcal{S}$. Soit

$$
\alpha: \mathfrak{X}_{G}\left(p^{n}, \mathcal{S}^{\prime}\right)^{\text {tor-mod }} \rightarrow \mathfrak{X}_{G}\left(p^{n}, \mathcal{S}\right)^{\text {tor-mod }}
$$

le morphisme induit. Il s'agit de voir que

$$
R \alpha_{\star} \mathscr{O}_{\mathfrak{X}_{G}\left(p^{n}, \mathcal{S}^{\prime}\right)^{\text {tor-mod }}}=\mathscr{O}_{\mathfrak{X}_{G}\left(p^{n}, \mathcal{S}\right)^{\text {tor-mod }}[0] .}[0
$$

Cela résulte du fait que les fibres de $\alpha$ sont des variétés rationnelles, ce qui se teste sur sur les fibres de $\mathfrak{X}\left(p^{n}, \mathcal{S}^{\prime}\right)^{\text {tor-mod }} \rightarrow \mathfrak{X}\left(p^{n}, \mathcal{S}\right)^{\text {tor-mod }}$. On a dans ce cas une description des compactification toroïdale localement pour la topologie étale (voir l'appendice) et on peut donc adapter les arguments de [H1], prop. 2.4.

Définissons maintenant l'action de $\mathbb{T}$. Soit $t \in \mathbb{T}$ une double classe qui définit donc une correspondance finie étale

$$
p_{1}, p_{2}: C \rightrightarrows X_{G}\left(p^{n}\right)
$$

Pour des choix combinatoires convenables, on a une compactification toroïdale $C^{\text {tor }}$ et des compactifications toroïdales $X_{G}\left(p^{n}\right)^{t o r, 1}$ et $X_{G}\left(p^{n}\right)^{t o r, 2}$ tels que les morphismes $p_{1}$ et $p_{2}$ s'étendent en un morphisme fini et plat $p_{1}: C^{\text {tor }} \rightarrow X_{G}\left(p^{n}\right)^{t o r, 1}$ et en un morphisme propre surjectif $p_{2}: C^{t o r} \rightarrow X_{G}\left(p^{n}\right)^{t o r, 2}$ ainsi qu'en des morphismes finis et plats $q_{1}, q_{2}$ : $C^{\star} \rightarrow X_{G}\left(p^{n}\right)^{\star}$. Appliquant les constructions fonctorielles de la partie 2, on obtient alors un diagramme commutatif où les notations sont évidentes :

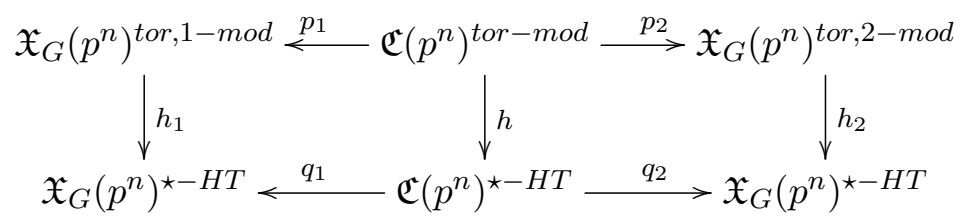

Définissons alors un morphisme

$$
t: \mathrm{H}^{\star}\left(\mathfrak{X}_{G}\left(p^{n}\right)^{\star-H T},\left(h_{2}\right)_{\star} \omega^{\kappa}\right) \longrightarrow \mathrm{H}^{\star}\left(\mathfrak{X}_{G}\left(p^{n}\right)^{\star-H T},\left(h_{1}\right)_{\star} \omega^{\kappa}\right) .
$$

On a pour commencer une application de restriction :

$$
\mathrm{H}^{\star}\left(\mathfrak{X}_{G}\left(p^{n}\right)^{\star-H T},\left(h_{2}\right)_{\star} \omega^{\kappa}\right) \rightarrow \mathrm{H}^{\star}\left(\mathfrak{C}\left(p^{n}\right)^{\star-H T}, q_{2}^{\star}\left(h_{2}\right)_{\star} \omega^{\kappa}\right) \rightarrow \mathrm{H}^{\star}\left(\mathfrak{C}\left(p^{n}\right)^{\star-H T}, h_{\star} p_{2}^{\star} \omega^{\kappa}\right) .
$$

D'autre part, on a sur $C^{\text {tor }}$ un morphisme $p_{2}^{\star} \omega^{\kappa} \rightarrow p_{1}^{\star} \omega^{\kappa}$ qui induit un morphisme analogue sur $\mathfrak{C}\left(p^{n}\right)^{\text {tor-mod }}$. On a donc une application

$$
\mathrm{H}^{\star}\left(\mathfrak{C}\left(p^{n}\right)^{\star-H T}, h_{\star} p_{2}^{\star} \omega^{\kappa}\right) \rightarrow \mathrm{H}^{\star}\left(\mathfrak{C}\left(p^{n}\right)^{\star-H T}, h_{\star} p_{1}^{\star} \omega^{\kappa}\right)=\mathrm{H}^{\star}\left(\mathfrak{X}_{G}\left(p^{n}\right)^{\star-H T},\left(q_{1}\right)_{\star} h_{\star} p_{1}^{\star} \omega^{\kappa}\right)
$$

Il nous reste donc a définir une trace

$$
\left(q_{1}\right)_{\star} h_{\star} p_{1}^{\star} \omega^{\kappa} \longrightarrow\left(h_{1}\right)_{\star} \omega^{\kappa}
$$

Comme $\left(q_{1}\right)_{\star} h_{\star}\left(p_{1}\right)^{\star} \omega^{\kappa}=\left(h_{1}\right)_{\star}\left(p_{1}\right)_{\star}\left(p_{1}\right)^{\star} \omega^{\kappa}$, il suffit de définir un morphisme

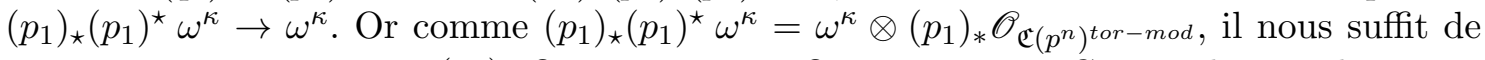

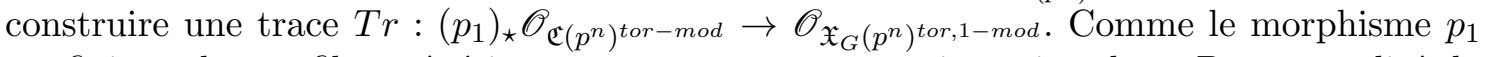
est fini et plat en fibre générique, on a une trace apres inversion de $p$. Par normalité, la trace respecte les structures entières d'après [S4, lem.IV.3.2]. 
Soit $r$ un entier et $\kappa$ une représentation irréductible de $M_{G}$. Nous noterons $\omega^{\kappa+r}$ le produit tensoriel de $\omega^{\kappa}$ par $(\omega \underline{\underline{1}})^{\otimes r}$. Soit $n$ un entier et $m=\sup \left\{n, n_{0}\right\}$. Pour tout entier $l \geq 0$, notons $\mathbb{T}(\kappa, n, l)$ l'image de $\mathbb{T}$ dans l'anneau des endomorphismes de

$$
\oplus_{r=0}^{l} S_{\kappa+r}\left(p^{m}\right) .
$$

C'est une $\mathbb{Z}_{p}$-algèbre libre de rang finie. Soit $\mathbb{T}^{p-a d}(\kappa, n)$ la limite projective sur $l$ des $\mathbb{T}(\kappa, n, l)$. On la munit de la topologie faible qui rend continue les projections $\mathbb{T}^{p-a d}(\kappa, n) \rightarrow$ $\mathbb{T}(\kappa, n, l)$ où $\mathbb{T}(\kappa, n, l)$ est équipée de la topologie $p$-adique.

Théorème 3.6. - L'action de $\mathbb{T}$ sur $S_{\kappa}^{i}\left(p^{n}\right)$ et sur $M_{\kappa}^{i}\left(p^{n}\right)$ se factorise par une action continue de $\mathbb{T}^{p-a d}(\kappa, n)$.

Démonstration. On se réduit à traiter le cas de $S_{\kappa}^{i}\left(p^{n}\right)$ par dualité de Serre. Comme $S_{\kappa}^{i}\left(p^{n}\right)$ est un facteur direct de $S_{\kappa}^{i}\left(p^{m}\right)$ si $m \geq n$, à cause de l'existence d'un morphisme trace, on peut supposer que $n \geq n_{0}$. D'après la proposition 3.5, il suffit donc de démontrer le résultat pour la représentation de $\mathbb{T}$ sur $\mathbb{S}_{\kappa}^{i}\left(p^{n}\right)=\mathrm{H}^{i}\left(\mathfrak{X}_{G}\left(p^{n}\right)^{\star-H T}, h_{\star} \omega^{\kappa}(-D)\right)$. Posons $\mathscr{F}:=h_{\star} \omega^{\kappa}(-D)$ et $\mathscr{L}:=\operatorname{det}^{k} \omega_{A}^{\text {mod }}$ pour alléger les notations. La cohomologie peut se calculer à l'aide du complexe de Cech associé au recouvrement

$$
\left\{\mathfrak{V}_{G, i}\left(p^{n}\right)=D\left(s_{i}^{k}\right)\right\}_{0 \leq i \leq r}
$$

où $D$ désigne l'ouvert de non annulation d'une section d'un fibré. Comme chaque ouvert du recouvrement est stable sous l'action des opérateurs de Hecke, il suffit de démontrer le résultat pour la représentation $\mathrm{H}^{0}\left(\mathfrak{V}_{G, I}\left(p^{n}\right), \mathscr{F}\right)$ de $\mathbb{T}$ où $I$ est un sous ensemble de $\{0, \ldots, r\}$ et $\mathfrak{V}_{G, I}\left(p^{n}\right)=\cap_{i \in I} \mathfrak{V}_{G, i}\left(p^{n}\right)$. Soit $s$ un entier positif. Il suffit de démontrer le résultat pour la représentation $\mathrm{H}^{0}\left(\mathfrak{V}_{G, I}\left(p^{n}\right), \mathscr{F} / p^{s}\right)$ de $\mathbb{T}$. Soit $T_{i} \in \mathrm{H}^{0}\left(\mathfrak{V}_{G, i}\left(p^{n}\right), \mathscr{L}\right)$ un relèvement de $t_{i}$ (voir la proposition 2.5). Soit $T_{I}=\prod_{i \in I} T_{i} \in \mathrm{H}^{0}\left(\mathfrak{V}_{G, I}\left(p^{n}\right), \mathscr{L}^{\otimes \sharp I}\right)$. Comme $T_{I}$ modulo $p^{n_{0}-\frac{g}{p-1}}$ (resp. $p^{n_{0}-2 g}$ si $p=2$ ) commute à l'action de $\mathbb{T}$, on a donc, pour $q$ suffisament grand, un isomorphisme équivariant sous Hecke

$$
\mathrm{H}^{0}\left(\mathfrak{V}_{G, I}\left(p^{n}\right), \mathscr{F} / p^{s}\right)=\operatorname{colim}_{u \geq 0} \mathrm{H}^{0}\left(\mathfrak{X}_{G}^{\star-H T}\left(p^{n}\right), \mathscr{F} / p^{s} \otimes \mathscr{L}^{\otimes \sharp I \cdot p^{u \cdot q}}\right)
$$

où les applications de transition sont données par la multiplication par $t_{I}^{p^{q}}$. Comme $\mathscr{L}$ est ample, pour $u$ suffisament grand on a

$$
\mathrm{H}^{0}\left(\mathfrak{X}_{G}^{\star-H T}\left(p^{n}\right), \mathscr{F} / p^{s} \otimes \mathscr{L}^{\otimes \sharp I \cdot p^{u \cdot q}}\right)=\mathrm{H}^{0}\left(\mathfrak{X}_{G}^{\star-H T}\left(p^{n}\right), \mathscr{F} \otimes \mathscr{L}^{\otimes \sharp I \cdot p^{u \cdot q}}\right) \otimes_{\mathbb{Z}} \mathbb{Z} / p^{s} \mathbb{Z} .
$$

Pour finir, remarquons que $\mathrm{H}^{0}\left(\mathfrak{X}_{G}^{\star-H T}\left(p^{n}\right), \mathscr{F} \otimes \mathscr{L}^{\otimes \sharp I \cdot p^{u \cdot q}}\right)[1 / p]=S_{\kappa+k \cdot \sharp I \cdot u \cdot q}\left(p^{n}\right)$.

Remarque 3.7. - L'algèbre $\mathbb{T}^{p-a d}(\kappa, n)$ du théorème est un quotient de l'agèbre $\mathbb{T}^{p-a d}$ $\mathrm{du}$ théorème principal énoncé dans l'introduction.

Remarque 3.8. - Pour $i=0$, le théorème précédent montre que les systèmes de valeurs propres de Hecke apparaissant dans l'espace des séries d'Eisenstein holomorphes apparaissent également dans l'espace des formes cuspidales.

Remarque 3.9. - Pour $i=0$, le théorème précédent est déjà intéressant pour $S_{\kappa}^{i}\left(p^{n}\right)$ lorsque $p$ est non ramifié mais pas totalement décomposé dans le corps réflexe car le lieu ordinaire de $X_{G}\left(p^{n}\right)$ est vide et les méthodes habituelles de multiplication par des relèvements de puissances de l'invariant de Hasse ne s'appliquent pas. Voir [GN].

Remarque 3.10. - On a en fait prouvé que l'action de $\mathbb{T}$ sur $\mathbb{S}_{\kappa}^{i}\left(p^{n}\right)$ se factorise par $\mathbb{T}^{p-a d}$ pour tout $n \geq n_{0}$ et $i \geq 0$. Cela est à mettre en perspective avec les résultats en cours de rédaction de Boxer et de Geraghty-Goldring, qui démontrent un analogue du théorème 3.6 dans certains cas PEL, mais en étudiant la structure entière $\mathbb{S}_{\kappa}^{\prime i}\left(p^{n}\right)$ 
sur $S_{\kappa}^{i}\left(p^{n}\right)$ provenant du modèle entier de Kottwitz. La torsion des structures entières $\mathbb{S}_{\kappa}^{i}\left(p^{n}\right)$ et $\mathbb{S}_{\kappa}^{\prime i}\left(p^{n}\right)$ semble être très différente et peut-être que l'une d'entre elles se revèlera plus pratique à manier. Remarquons enfin que la problématique de Boxer est opposée à la nôtre puisqu'il déduit de ses résultats une nouvelle démonstration de certains cas du théorème principal de $[\mathbf{S 4}]$.

3.11. Représentations galoisiennes. - Le résultats du paragraphe précédent est spécialement intéressant lorsqu'on sait que les systèmes de valeurs propres de $\mathbb{T}^{p-a d}$ sont associés à des représentations galoisiennes. C'est le cas lorsque $G$ est égal à $\mathrm{GSp}_{4}$ ([L2], $[\mathbf{T}]$ et $[\mathbf{W}]), \mathrm{Sp}_{2 g}$ sur un corps totalement réel $[\mathbf{A}]$ ou un groupe unitaire sur un corps $\mathrm{CM}$ ([Mo] et $[\mathbf{K M S W}]$ ), ces trois dernières références étant conditionnelles à la stabilisation de la formule des traces tordue (mais voir les travaux récents de Waldspurger et Moeglin). On s'attend de plus à ce que ce soit le cas lorsque $G=\mathrm{GSp}_{2 g}$ sur un corps totalement réel (voir $[\mathbf{X}]$ ).

3.11.1. Cas Siegel-Hilbert. - Formulons le résultat obtenu dans le cas où $G=\mathrm{Sp}_{2 g}$ sur un corps totalement réel $F$, qui concerne donc les variétés de Hilbert-Siegel connexes. Soit

$$
\eta:{ }^{L} \mathrm{G} \longrightarrow \mathrm{GL}_{2 g+1} / F
$$

la représentation standard du groupe dual sur $F$ obtenue grâce à la projection

$$
\operatorname{Spin}(2 n+1) \longrightarrow \mathrm{SO}(2 n+1) .
$$

Pour toute place finie $v$ de $F$ non ramifiée sur $\mathbb{Q}$, on rappelle l'isomorphisme de Satake pour l'algèbre de Hecke $\mathbb{T}_{v}$ non ramifiée en $v$

$$
\mathbb{T}_{v}\left[q_{v}^{\frac{1}{2}}\right] \simeq \mathbb{Z}\left[q_{v}^{\frac{1}{2}}\right]\left[Y\left(\mathrm{~T}_{\mathrm{Sp}_{2 g}}\right)\right]^{W_{\mathrm{Sp}_{2 g}}}
$$

où $q_{v}$ est le cardinal du corps résiduel de $v$, où $\mathrm{T}_{\mathrm{Sp}_{2 g}}=\mathbb{G}_{m}^{g}$ est le tore maximal standard de $\mathrm{G}$, où $Y$ désigne le groupe des cocaractères et où $W$ est le groupe de Weyl. De même pour $\mathrm{GL}_{2 g+1}$. L'application $\eta$ induit un morphisme

$$
\eta_{v}: \mathbb{Z}\left[q_{v}^{\frac{1}{2}}\right]\left[Y\left(\mathrm{~T}_{\mathrm{GL}_{2 \mathrm{~g}+1}}\right)\right]^{W_{\mathrm{GL}_{2 g+1}}} \longrightarrow \mathbb{Z}\left[q_{v}^{\frac{1}{2}}\right]\left[Y\left(\mathrm{~T}_{\mathrm{Sp}_{2 g}}\right)\right]^{W_{\mathrm{Sp}_{2 g}}} .
$$

Pour $1 \leq i \leq 2 g+1$, soit $T_{i, v}$ l'image par $\eta_{v}$ de la $i$-ème fonction symétrique en la base standard de $Y\left(\mathrm{~T}_{\mathrm{GL}_{2 \mathrm{~g}+1}}\right)$. On note $\mathbb{T}^{S}=\otimes_{v \notin S} \mathbb{T}_{v}$ dès que cela a un sens.

Corollaire 3.12. - Soit $\pi$ une représentation automorphe pour $G$ de niveau premier $\grave{a}$ un ensemble fini $S$ de places de $F$ contenant les places ramifiées au-dessus de $\mathbb{Q}$. On suppose que $\pi$ apparaît dans la cohomologie cohérente de la variété de Siegel-Hilbert de genre $g$ pour $F$ et de niveau convenable. Soit $\Theta_{\pi}: \mathbb{T}^{S} \rightarrow \mathbb{E}$ le système de valeur propre associé, à valeur dans un corps de nombres $\mathbb{E}$. Alors pour toute place $\lambda$ de $\mathbb{E}$ au dessus d'une place $\ell$ de $\mathbb{Q}$, il existe une représentation du groupe de Galois $G_{F, S \ell}$ de l'extension maximale de $F$ non ramifiée hors de $S \cup\{w \mid \ell\}$ :

$$
\rho_{\pi, \lambda}: G_{F, S \ell} \rightarrow \mathrm{GL}_{2 g+1}\left(\mathbb{E}_{\lambda}\right)
$$

qui est semi-simple, continue telle que $\rho_{\pi, \lambda}^{\vee} \simeq \rho_{\pi, \lambda}$ et que pour toute place $v \notin S \cup\{w \mid \ell\}$, $\operatorname{det}\left(1-\mathrm{Frob}_{v} \cdot X \mid \rho_{\pi, \lambda}\right)=\Theta_{\pi}\left(1-T_{1, v} \cdot X+T_{2, v} \cdot X^{2}+\ldots+(-1)^{2 g+1} T_{2 g+1, v} \cdot X^{2 g+1}\right)$. 
3.12.1. Cas unitaire quasi-déployé. - Soit $G$ un groupe unitaire quasi-déployé associé à $F / F^{+}$un corps CM. On se place dans le cas où $G$ est forme de $\mathrm{GL}_{2 g} / F$. Le cas d'une forme de $\mathrm{GL}_{2 g+1} / F$ marcherait tout aussi bien avec des énoncés légèrement adaptés. On choisit le plongement standard

$$
\eta:{ }^{L} \mathrm{G} \longrightarrow{ }^{L}\left(\operatorname{Res}_{F / F^{+}}\left(\mathrm{GL}_{2 g} / F\right)\right)
$$

de groupe dual sur $F^{+}$, où $G$ est une forme de $\mathrm{GL}_{2 g}$. La forme de l'isomorphisme de Satake dépendant du comportement des nombres premiers dans $F$, discutons-là cas par cas.

Soit $v$ une place de $F^{+}$de cardinal résiduel $q_{v}$ non ramifiée sur $\mathbb{Q}$ et décomposée dans $F$. Soit $\tilde{v}$ une place de $F$ au dessus de $v$. On a $G\left(F_{v}^{+}\right) \simeq \mathrm{GL}_{2 g}\left(F_{\tilde{v}}\right)$ l'isomorphisme de Satake est alors

$$
\mathbb{T}_{v}\left[q_{v}^{\frac{1}{2}}\right] \simeq \mathbb{Z}\left[q_{v}^{\frac{1}{2}}\right]\left[Y\left(\mathrm{~T}_{\mathrm{GL}_{2 g}}\right)\right]^{W_{\mathrm{GL}_{2 g}}}
$$

qui induit un morphisme $\eta_{v}$ s'identifiant à l'identité de $\mathbb{Z}\left[q_{v}^{\frac{1}{2}}\right]\left[Y\left(\mathrm{~T}_{\mathrm{GL}_{2 \mathrm{~g}}}\right)\right]^{W_{\mathrm{GL}_{2 g}}}$ et l'on note $T_{i, \tilde{v}}$ la $i$-ème fonction symétrique en la base standard de $Y\left(\mathrm{~T}_{\mathrm{GL}_{2 \mathrm{~g}}}\right)$ pour $1 \leq i \leq 2 g$.

Soit $v$ une place de $F^{+}$non ramifiée sur $\mathbb{Q}$ et inerte dans $F$. Soit $B$ un sous-groupe de Borel défini sur $F^{+}$, soit $\mathrm{T}_{G, d}$ un tore maximal décomposé contenu dans $B$ et soit $W_{G, d}$ le sous-groupe du groupe de Weyl qui agit sur le tore $\mathrm{T}_{G, d}$. Un tel tore est isomorphe à $\mathbb{G}_{m}^{g}$, le groupe $W_{d}$ est isomorphe à $\mathcal{S}_{g} \ltimes(\mathbb{Z} / 2 \mathbb{Z})^{g}$ et l'action de $W_{d}=\mathcal{S}_{g} \ltimes(\mathbb{Z} / 2 \mathbb{Z})^{g}$ sur $\mathrm{T}_{G, d}=\mathbb{G}_{m}^{g}$ est l'action évidente. L'isomorphisme de Satake est

$$
\mathbb{T}_{v}\left[q_{v}^{\frac{1}{2}}\right] \simeq \mathbb{Z}\left[q_{v}^{\frac{1}{2}}\right]\left[Y\left(\mathrm{~T}_{G, d}\right)\right]^{W_{d}} .
$$

Le morphisme $\eta$ induit alors un morphisme

$$
\eta_{v}: \mathbb{Z}\left[q_{v}^{\frac{1}{2}}\right]\left[Y\left(\mathrm{~T}_{\mathrm{GL}_{2 \mathrm{~g}}}\right)\right]^{W_{\mathrm{GL}_{2 g}}} \longrightarrow \mathbb{Z}\left[q_{v}^{\frac{1}{2}}\right]\left[Y\left(\mathrm{~T}_{G, d}\right)\right]^{W_{d}} .
$$

On note encore $T_{i, v}$ la $i$-ème fonction symétrique en la base standard de $Y\left(\mathrm{~T}_{\mathrm{GL}_{2 \mathrm{~g}}}\right)$ pour $1 \leq i \leq 2 g$. Soit $\chi_{\ell}$ le caractère cyclotomique $\ell$-adique et $c$ le générateur du groupe de Galois de $F / F^{+}$.

Corollaire 3.13. - Soit $\pi$ une représentation automorphe pour $G$ qui apparaît dans la cohomologie cohérente de la variété de Shimura unitaire connexe associée à $G$ et de niveau premier à un ensemble fini $S^{+}$de places de $F^{+}$. Soit $S$ l'ensemble fini de places de $F$ au dessus d'une place de $S^{+}$. Soit $\Theta_{\pi}: \mathbb{T}^{S} \rightarrow \mathbb{E}$ le système de valeurs propres de $\pi$ qui est à valeur dans un corps de nombre $\mathbb{E}$. Pour toute place $\lambda$ de $E$ au dessus d'un nombre premier $\ell$ de $\mathbb{Q}$, il existe une représentation du groupe de Galois $G_{F, S \ell}$ de l'extension maximale de $F$ non ramifiée hors de $S \cup\{w \mid \ell\}$ :

$$
\rho_{\pi, \lambda}: G_{F, S \ell} \longrightarrow \mathrm{GL}_{2 g}\left(\mathbb{E}_{\lambda}\right)
$$

qui est semi-simple, continue telle que $\left(\rho_{\pi, \lambda}^{\vee}\right)^{c} \simeq \rho_{\pi, \lambda} \otimes \chi_{\ell}$ et que pour toute place $v \notin$ $S \cup\{w \mid \ell\}$ de cardinal résiduel $q_{v}$, on ait

$\operatorname{det}\left(1-\mathrm{Frob}_{v} \cdot X \mid \rho_{\pi, \lambda}\right)=\Theta_{\pi}\left(1-q_{v}^{\frac{1}{2}} \cdot T_{1, v} \cdot X+q_{v}^{\frac{2}{2}} \cdot T_{2, v} \cdot X^{2}+\ldots+(-1)^{2 g} q_{v}^{\frac{2 g}{2}} \cdot T_{2 g, v} \cdot X^{2 g}\right)$.

3.13.1. Cas $\mathrm{GSp}_{4}$. - Supposons que $G$ est le groupe $\mathrm{GSp}_{4}$ sur $\mathbb{Q}$. Le réseau $Y\left(T_{\mathrm{GSp}_{4}}\right)$ s'identifie à l'ensemble des entiers relatifs $(a, b, c, d)$ tels que $a+c=b+d$. Le groupe de Weil est $\mathfrak{S}_{2} \ltimes(\mathbb{Z} / 2)^{2}$ où l'élément non trivial du premier facteur agit par $(a, b, c, d) \mapsto(b, a, d, c)$, le premier facteur $(\mathbb{Z} / 2)^{2}$ agit par $a \leftrightarrow c$ et le second par $b \leftrightarrow d$. L'isomorphisme de Satake est pour toute place $v$ de $\mathbb{Q}$

$$
\mathbb{T}_{v}\left[q_{v}^{\frac{1}{2}}\right] \simeq \mathbb{Z}\left[q_{v}^{\frac{1}{2}}\right]\left[Y\left(\mathrm{~T}_{\mathrm{GSp}_{4}}\right)\right]^{W_{\mathrm{GS}_{4}}}
$$


On considère le plongement canonique $\eta: G=\hat{G} \hookrightarrow \mathrm{GL}_{4}$ qui induit

$$
\eta_{v}: \mathbb{Z}\left[q_{v}^{\frac{1}{2}}\right]\left[Y\left(\mathrm{~T}_{\mathrm{GL}_{4}}\right)\right]^{W_{\mathrm{GL}_{4}}} \longrightarrow \mathbb{Z}\left[q_{v}^{\frac{1}{2}}\right]\left[Y\left(\mathrm{~T}_{\mathrm{GSp}_{4}}\right)\right]^{W_{\mathrm{GSp}_{4}}} .
$$

Soit $T_{i, v}$ l'image de la $i$-ème fonction symétrique de la base canonique de $Y_{\mathrm{GL}_{4}}$ pour tout $1 \leq i \leq 4$.

Corollaire 3.14. - Soit $\pi=\otimes_{v} \pi_{v}$ une représentation automorphe pour $\mathrm{G}$ de niveau premier à un ensemble fini $S$ qui apparaît dans la cohomologie cohérente d'une variété de Siegel de genre 2 . Soit $\Theta_{\pi}: \mathbb{T}^{S} \rightarrow \mathbb{E}$ le système de valeur propre associé à valeur dans un corps de nombres $\mathbb{E}$. Pour toute place $\lambda$ de $\mathbb{E}$ au dessus d'un nombre premier $\ell$, il existe une représentation $\rho_{\pi, \lambda}: G_{\mathbb{Q}, S \ell} \rightarrow \mathrm{GL}_{4}\left(\mathbb{E}_{\lambda}\right)$ du groupe de Galois $G_{\mathbb{Q}, \text { Sl }}$ de l'extension maximale de $\mathbb{Q}$ non ramifiée hors de $S \cup\{\ell\}$ qui est semi-simple continue telle que pour toute place $v \notin S \cup\{\ell\}$, on ait $\operatorname{det}\left(1-\right.$ Frob $\left._{v} \cdot X \mid \rho_{\pi, \lambda}\right)=$

$$
\Theta_{\pi}\left(1-q_{v}^{\frac{3}{2}} \cdot T_{1, v} \cdot X+\left(q_{v}^{\frac{3}{2}}\right)^{2} \cdot T_{2, v} \cdot X^{2}-\left(q_{v}^{\frac{3}{2}}\right)^{3} \cdot T_{3, v} \cdot X^{3}+\left(q_{v}^{\frac{3}{2}}\right)^{4} \cdot T_{4, v} \cdot X^{4}\right) .
$$

3.15. Limites de séries discrètes. - Il reste à quantifier quelles représentations automorphes interviennent dans la cohomologie cohérente des variétés de Shimura, et spécialement celles qui n'interviennent pas dans la cohomologie étale ou dans le $H^{0}$ cohérent. Les résultats dont on dispose reposent sur la classification des représentations admissibles des groupes réels et le calcul de leur $\left(\mathfrak{p}, K_{\infty}\right)$-cohomologie. L'article [H3] présente un panorama de la théorie. Contentons-nous de rappeler deux résultats fondamentaux :

1. Les représentations automorphes $\pi=\pi_{\infty} \otimes \pi_{f}$ de $G(\mathbb{A})$ telles que $\pi_{\infty}$ est limite non dégénérée de séries discrètes (modulo le centre) contribuent à la cohomologie cohérente de $X_{G}$ ([H3, th.3.4]). De plus, $\pi$ intervient dans la cohomologie étale lorsque $\pi_{\infty}$ est série discrète et dans le $\mathrm{H}^{0}$ cohérent lorsque $\pi_{\infty}$ est limite holomorphe de séries discrètes.

2. Réciproquement, si $\pi_{\infty}$ est tempérée, et $\pi$ contribue à la cohomologie cohérente, alors $\pi_{\infty}$ est limite non dégénérée de séries discrètes ([H3, th.3.5]).

Illustrons maintenant la théorie par quelques exemples.

3.15.1. Le groupe $\mathrm{Sp}_{2 g} / \mathbb{Q}$. - Soit $\mathrm{T} \simeq \mathbb{G}_{m}^{g}$ le tore maximal standard de $G=\mathrm{Sp}_{2 g}$. Un caractère du tore est noté $\left(a_{1}, \ldots, a_{g}\right) \in \mathbb{Z}^{g}$. On note $\alpha_{i}$ le caractère correspondant au vecteur de $\mathbb{Z}^{g}$ qui a une seule coordonnée non nulle égale à 1 en position $i$. On adopte le système de racine positive $R^{+}$formé par les racines non-compactes $R_{n c}^{+}=\left\{\alpha_{i}+\alpha_{j}, 1 \leq\right.$ $i \leq j \leq g\}$ et les racines compactes $R_{c}^{+}=\left\{\alpha_{i}-\alpha_{j}, 1 \leq i<j \leq g\right\}$. La demi-somme des racines positives vaut $\rho=(g, g-1, \ldots, 1)$. La demi-somme des racines compactes vaut $\rho_{c}=\frac{1}{2}(g-1, g-3, \ldots, 1-g)$ et la demi-somme des racines positives non-compactes vaut $\rho_{n c}=\frac{1}{2}(g+1, \ldots, g+1)$. Un poids $\kappa=\left(a_{1}, \ldots, a_{g}\right)$ est dominant pour $R_{c}^{+}$si $a_{1} \geq a_{2} \ldots \geq a_{g}$ et il lui correspond un faisceau localement libre $\omega^{\kappa}$ sur la variété de Siegel. Au poids $\kappa=\left(a_{1}, \ldots, a_{g}\right)$ on associe le caractère dominant et régulier pour $R_{c}^{+}, \lambda(\kappa)=\kappa+\rho_{c}-\rho_{n c}=$ $\left(a_{1}-1, a_{2}-2, \ldots ., a_{g}-g\right)$. Soit $\lambda=\left(\lambda_{1}, \ldots ., \lambda_{g}\right)$ un caractère dominant et régulier pour $R_{c}^{+}$ (c'est à dire $\lambda_{1}>\ldots .>\lambda_{g}$ ) et soit $C$ un système de racine positif telle que $R_{c}^{+} \subset C$ et telle que $\lambda$ soit dominant pour $C$. Remarquons que si $\lambda$ est de plus régulier pour $R^{+}$(c'est à dire $\left.\lambda_{i} \neq-\lambda_{j}\right)$, alors $C$ est uniquement déterminé par $\lambda$. Au couple $(\lambda, C)$ est associé une représentation $\pi(\lambda, C)$ du groupe $\mathrm{Sp}_{2 g}(\mathbb{R})$. Lorsque $\lambda$ est régulier pour $R^{+}$, c'est la série discrète de paramètre $(\lambda, C)$ (ou simplement $\lambda$ ) et lorsque $\lambda$ n'est pas régulier pour $R_{n c}^{+}$ c'est la limite non-dégénérée de série discrète de paramètre $(\lambda, C)$. Cette représentation a pour caractère infinitésimal $\lambda$. 
Soit $\pi$ une représentation automorphe de $G(\mathbb{A})$ telle que $\pi_{\infty}$ soit une série discrète ou une limite non dégénérée de série discrète de paramètre $(\lambda, C)$. Alors $\pi$ contribue à la cohomologie de $\omega^{\kappa}$ si et seulement si $\lambda=\lambda(\kappa)$. De plus elle contribue en degré $r$ si il existe un représentant de Kostant $w$ tel que $w \lambda$ soit dominant pour $R^{+}$et $\ell(w)=r$. Si $\lambda$ est régulier pour $R^{+}$alors $r$ est uniquement déterminé par $\lambda$, en revanche, si $\lambda$ n'est pas régulier pour $R_{n c}^{+}$, alors $\pi$ contribue en degré $r_{0}, r_{0}+1, \ldots, r_{0}+\ell_{0}$ où $\ell_{0}$ est égal au nombre de racines positives non-compactes $\alpha$ telles que $\langle\alpha, \lambda\rangle=0$ (ou au nombre de murs de la chambre de Weyl associé à $C$ que rencontre $\lambda$ ).

Par exemple si $\lambda_{1}>\ldots>\lambda_{g}>0$, la représentation contribue uniquement au $\mathrm{H}^{0} \mathrm{du}$ faisceau $\omega^{\kappa}$ avec $\kappa=\left(\lambda_{1}-1, \ldots ., \lambda_{g}-g\right)$. Si on a $g=2 r, \lambda=\left(\lambda_{1}, \ldots ., \lambda_{r},-\lambda_{r}, \ldots,-\lambda_{1}\right)$ avec $\lambda_{1}>\lambda_{2} \ldots>\lambda_{r}>0$, alors la représentation contribue en $r$ degrés de cohomologie (centré autours de la dimension moitié) du faisceau $\omega^{\kappa}$.

Si on considère la représentation $\eta:{ }^{L} G \rightarrow \mathrm{GL}_{2 g+1}$ du groupe dual, alors pour $\pi$ qui est limite non-dégénérée de série discrète, on a un système compatible de représentations $\rho_{\pi, \lambda}: G_{\mathbb{Q}} \rightarrow \mathrm{GL}_{2 g+1}\left(\mathrm{E}_{\lambda}\right)$. Si le paramètre $(\lambda, C)$ de $\pi_{\infty}$ est régulier pour $R^{+}$(et $\pi_{\infty}$ est une série discrète), alors $\rho_{\pi, \lambda}$ a pour poids de Hodge-Tate $\left( \pm \lambda_{1}, \ldots, \pm \lambda_{g}, 0\right)$. Il est naturel de conjecturer que c'est le cas en général. Dans le cas où la représentation contribue au $\mathrm{H}^{0}$ de la cohomologie cohérente, on peut le montrer en utilisant la variété de Hecke.

3.15.2. Groupes unitaires. - Soit $F / \mathbb{Q}$ une extension quadratique imaginaire et $g=$ $p+q$ des entiers. Soit $G$ un groupe unitaire sur $\mathbb{Q}$ associé à une forme hermitienne sur $F$ de signature $(p, q)$. Soit $\pi$ une représentation automorphe de $G(\mathbb{A})$ de composante à l'infini $\pi_{\infty}$. On dispose alors du paramètre de Harish-Chandra $\chi_{\pi_{\infty}}$ de $\pi_{\infty}$ qui s'identifie à un $g$-uplet d'entiers $\left(\lambda_{1}, \lambda_{2}, \cdots, \lambda_{g}\right)$. La normalisation est choisie pour que, lorsque $\pi_{\infty}$ est série discrète, on ait $\lambda_{1}>\cdots>\lambda_{g}$ et les poids de Hodge-Tate de la représentation galoisienne $\rho_{\pi}$ associée à $\pi$ par Kottwitz, Clozel, Harris, Taylor, Shin et Chenevier (en tenant compte de $\left[\mathbf{G}\right.$, app.A] dû à Shin) soient $\lambda_{1}>\cdots>\lambda_{g}$. Ce cas où $\pi_{\infty}$ est série discrète est donc caractérisé par le fait que les poids de Hodge-Tate de $\rho_{\pi}$ sont tous distincts. On remarque que la signature $(p, q)$ n'influe pas sur les poids de Hodge-Tate possibles.

La composante à l'infini $\pi_{\infty}$ est limite holomorphe de séries discrètes si et seulement si on a $\lambda_{1}>\cdots>\lambda_{p} \geq \lambda_{p+1}>\cdots>\lambda_{g}$. Rappelons que dans ce cas, $\pi_{f}$ intervient dans le $\mathrm{H}^{0}$ cohérent. On s'attend alors que les poids de Hodge-Tate de la représentation obtenue dans $[\mathbf{G}]$ ou dans la partie 3.12 .1 soient $\lambda_{1}>\cdots>\lambda_{p} \geq \lambda_{p+1}>\cdots>\lambda_{g}$ et aient donc au plus une répétition dont la position dépend de la signature. Dans le cas où $p=q=1$ cela résulte des travaux de Deligne et Serre. Dans le cas général où le lieu ordinaire est non vide, on peut le montrer en effectuant un changement de base pour rendre $\pi_{p}$ de pente finie puis utiliser la théorie des variétés de Hecke.

La composante à l'infini $\pi_{\infty}$ est limite non dégénérée de séries discrètes si et seulement si on a $\lambda_{1}>\cdots>\lambda_{p}$ et $\lambda_{p+1}>\cdots>\lambda_{g}$. La représentation $\pi_{f}$ intervient alors dans le $\mathrm{H}^{q}$ cohérent où $q$ est le nombre minimal de transpositions élémentaires du type $(i, i+1)$ nécessaires pour classer la liste $\left(\lambda_{1}, \cdots, \lambda_{g}\right)$. On conjecture alors que les poids de Hodge-Tate de la représentation galoisienne $\rho_{\pi}$ construite dans la partie 3.12 .1 sont toujours $\lambda_{1}, \cdots, \lambda_{g}$ et que chaque poids apparaît donc avec multiplicité au plus deux. Le nombre de poids multiples est égal à $\min (p, q)$. La théorie est donc censée être la plus riche dans le cas quasi-déployé.

3.15.3. Le groupe $\mathrm{GSp}_{4}$. - La théorie des limites non-dégénérée de série discrète se déduit de celle pour $\mathrm{Sp}_{4}$. Le tore maximal de $\mathrm{GSp}_{4}$ est le quotient du produit du tore maximal de $\mathrm{Sp}_{4}$ et du centre par un groupe d'ordre 2 . Les caractères du tore sont paramétrés par des triplets $\left(a_{1}, a_{2} ; c\right) \in \mathbb{Z}^{3}$ avec $c=a_{1}+a_{2} \quad \bmod 2$. Le couple $\left(a_{1}, a_{2}\right)$ est un caractère du tore 
du groupe dérivé et $c$ le caractère du centre. Soit $w$ l'élément du groupe de Weyl qui envoit un caractère $\left(a_{1}, a_{2} ; c\right)$ sur $\left(-a_{2},-a_{1} ; c\right)$. Les limites non dégénérées de série discrètes sont paramétrées par les couples formés d'un caractère $\lambda=\left(\lambda_{1}, \lambda_{2} ; c\right)$ avec $\lambda_{1}>\lambda_{2} \geq-\lambda_{1}$, et d'un système de racine $C$ qui contient la racine compact et qui rend $\lambda$ dominant (il y a donc au plus 2 choix pour $C$ ). La restriction à $\operatorname{Sp}_{4}(\mathbb{R})$ de la limite non-dégénérée de série discrète de paramètre $(\lambda, C)$ est la somme directe des limites non-dégénérée de série discrète de paramètre $(\lambda, C)$ et $w(\lambda, C)$. Soit $\pi$ un forme automorphe pour $G$ avec $\pi_{\infty}$ série discrète de paramètre $(\lambda, C)$. Pour $\lambda_{1}>\lambda_{2}>0$ on obtient une série discrète qui apparaît dans le $\mathrm{H}^{0}$ du faisceau $\omega^{\kappa}$ avec $\kappa=\left(\lambda_{1}-2, \lambda_{2}-1 ; c\right)$ et dans le $\mathrm{H}^{3}$ du faisceau $\left(\omega^{\kappa}\right)^{\vee}$ (dual de Serre). Lorsque $\lambda_{2}=0$ on obtient une limite holomorphe de série discrète qui apparaît maintenant dans $\mathrm{H}^{0}$ et $\mathrm{H}^{1}$ de $\omega^{\kappa}$ et $\mathrm{H}^{2}$ et $\mathrm{H}^{3}$ de $\left(\omega^{\kappa}\right)^{\vee}$. Pour $\lambda_{1}>0>\lambda_{2}>-\lambda_{1}$, on obtient de nouveau une série discrète qui cette fois apparait dans le $\mathrm{H}^{1}$ de $\omega^{\kappa}$ avec $\kappa=\left(\lambda_{1}-2, \lambda_{2}-1 ; c\right)$ et le $\mathrm{H}^{2}$ de $\left(\omega^{\kappa}\right)^{\vee}$. Lorsque $\lambda_{1}=-\lambda_{2}$ on a une limite non dégénérée de série discrète qui apparaît maintenant dans le $\mathrm{H}^{1}$ et le $\mathrm{H}^{2}$ des faisceau $\omega^{\kappa}$ et $\left(\omega^{\kappa}\right)^{\vee}$. Si on note $\rho_{\pi, \lambda}$ la représentation à valeur dans $\mathrm{GL}_{4}$ associée à $\pi$, alors les poids de Hodge-Tate doivent être (à un twist à la Tate près), $\left(\lambda_{1}+\lambda_{2}, \lambda_{1}, \lambda_{2}, 0\right)$. Ceci est connu sauf dans le cas où $\lambda_{2}=-\lambda_{1}$.

\section{Appendice A}

\section{Compactifications}

Dans cet appendice, nous introduisons des compactifications toroïdales et minimales entières des variétés de Siegel avec un niveau plein $p^{n}$ en $p$ et nous décrivons le bord de ces compactifications. Ceci nous permet de justifier le fait que les formes modulaires associées aux classes de Hodge-Tate s'étendent à la compactification toroïdale et que les produits extérieurs $g$ à $g$ descendent à la minimale. Nous montrons également que les compactifications toroïdales des variétés de Siegel en niveau $p^{\infty}$ sont perfectoïdes. Pour simplifier la rédaction, nous faisons comme si $K^{p}=\mathrm{GSp}_{2 g}\left(\prod_{q \neq p} \mathbb{Z}_{q}\right)$.

A.1. Les cartes formelles. - Commençons par introduire les cartes formelles qui permettent de décrire le bord des compactifications torö̈dales des variétés de Siegel. Soit $V=\oplus_{i=1}^{2 g} \mathbb{Z} e_{i}$ un $\mathbb{Z}$-module libre de rang $2 g$ équipé d'une forme symplectique de matrice

$$
J=\left(\begin{array}{cc}
0 & 1_{g} \\
-1_{g} & 0
\end{array}\right)
$$

Pour tout facteur direct totalement isotrope $V^{\prime}$ de $V$ on considère $C\left(V / V^{\prime \perp}\right)$ le cône des formes bilinéaires symétriques semi-définie positives sur $\left(V / V^{\perp \perp}\right) \otimes \mathbb{R}$ dont le noyau est défini sur $\mathbb{Q}$. Si $V^{\prime} \subset V^{\prime \prime}$ on a une inclusion $C\left(V / V^{\prime \prime}\right) \subset C\left(V / V^{\perp}\right)$. Soit $\mathfrak{C}$ l'ensemble de tous les facteurs directs totalement isotropes $V^{\prime} \subset V$ et $\mathcal{C}$ le quotient de l'union disjointe

$$
\coprod_{V^{\prime} \in \mathfrak{C}} C\left(V / V^{\perp}\right)
$$

par la relation d'équivalence induite par les inclusions $C\left(V / V^{\prime \prime}\right) \subset C\left(V / V^{\perp \perp}\right)$. Soit $\Gamma=$ $\operatorname{GSp}(V)$. Fixons $\mathcal{S}$ une décomposition polyhédrale rationelle de $\mathcal{C}$ qui est $\Gamma$-admissible (voir [FC], Chap IV, sect. 2). Soit $V^{\prime} \in \mathfrak{C}$ un module de rang $r$ et $\sigma \in \mathcal{S}$ dans l'intérieur 
de $C\left(V / V^{\prime \perp}\right)$. On va construire pour tout $n \geq 1$ un diagramme

(A.1.A)

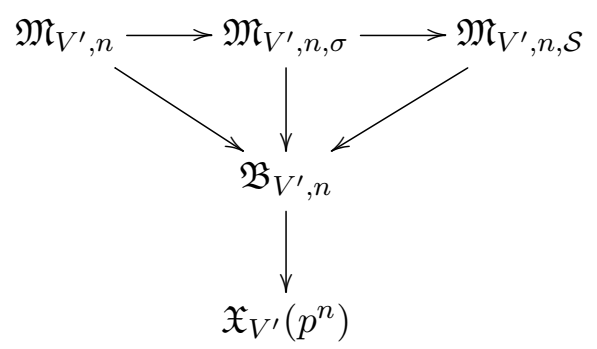

Les objets de ces diagrammes sont définis ainsi :

- $\mathfrak{X}_{V^{\prime}} \rightarrow \operatorname{Spf}\left(\mathcal{O}_{\mathbb{C}_{p}}\right)$ est la variété de Siegel formelle de genre $g-r$, où $r=\operatorname{rg}_{\mathbb{Z}}\left(V^{\prime}\right)$. On note $A_{V^{\prime}}$ le schéma abélien universel sur $\mathfrak{X}_{V^{\prime}}$. Soit $\mathcal{X}_{V^{\prime}}$ la fibre générique rigide de $\mathfrak{X}_{V^{\prime}}$. On note $\mathcal{X}_{V^{\prime}}\left(p^{n}\right) \rightarrow \mathcal{X}_{V^{\prime}}$ le revêtement étale qui paramètre des isomorphismes symplectiques $A_{V^{\prime}}\left[p^{n}\right] \simeq\left(V^{\prime \perp} / V^{\prime}\right) \otimes_{\mathbb{Z}} \mathbb{Z} / p^{n} \mathbb{Z}$. On définit $\mathfrak{X}_{V^{\prime}}\left(p^{n}\right) \rightarrow \mathfrak{X}_{V^{\prime}}$ comme la normalisation de $\mathfrak{X}_{V^{\prime}}$ dans $\mathcal{X}_{V^{\prime}}\left(p^{n}\right)$.

- On note

$$
\mathfrak{B}_{V^{\prime}, n}=\operatorname{Hom}_{\mathfrak{X}_{V^{\prime}}\left(p^{n}\right)}\left(\frac{1}{p^{n}} V / V^{\prime \perp}, A_{V^{\prime}}\right) \simeq A_{V^{\prime}}^{r} \times_{\mathfrak{X}_{V^{\prime}}} \mathfrak{X}_{V^{\prime}}\left(p^{n}\right)
$$

Au dessus de $\mathfrak{B}_{V^{\prime}}=\mathfrak{B}_{V^{\prime}, 0}$, on a un morphisme universel $c: V / V^{\prime \perp} \rightarrow A_{V^{\prime}}$ et un schéma semi-abelien universel

$$
0 \longrightarrow \mathrm{T}_{V^{\prime}} \longrightarrow \tilde{G}_{V^{\prime}} \longrightarrow A_{V^{\prime}} \rightarrow 0
$$

où $\mathrm{T}_{V^{\prime}}$ est le tore $V^{\prime} \otimes_{\mathbb{Z}} \mathbb{G}_{m}$. Au dessus de $\mathfrak{B}_{V^{\prime}, n}$, on a un morphisme universel

$$
c_{n}: \frac{1}{p^{n}} V / V^{\prime \perp} \longrightarrow A_{V^{\prime}}
$$

et une isogénie de schémas semi-abéliens

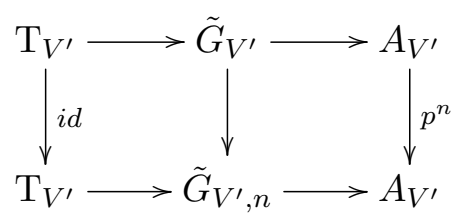

de noyau $A_{V^{\prime}}\left[p^{n}\right] \hookrightarrow \tilde{G}_{V^{\prime}}\left[p^{n}\right]$.

- $\mathfrak{M}_{V^{\prime}}=\mathfrak{M}_{V^{\prime}, 0}$ est la complétion formelle $p$-adique de l'espace de modules des 1-motifs principalement polarisés. On peut décrire cet espace de modules ainsi : il paramètre les trivialisations bilinéaires symétriques au dessus de $V / V^{\perp} \times V / V^{\perp}$ du fibré $(c \times c)^{\star} \mathcal{P}^{-1}$ où $\mathcal{P} \rightarrow A_{V^{\prime}} \times A_{V^{\prime}}$ est la bi-extension de Poincaré. Au dessus de $\mathfrak{M}_{V^{\prime}}$, on a un 1 -motif principalement polarisé universel

$$
M_{V^{\prime}}=\left[V / V^{\prime \perp} \longrightarrow \tilde{G}_{V^{\prime}}\right] .
$$

L'espace $\mathfrak{M}_{V^{\prime}, n}$ paramètre, au dessus de $\mathfrak{M}_{V^{\prime}} \times_{\mathfrak{X}_{V^{\prime}}} \mathfrak{X}_{V^{\prime}}\left(p^{n}\right)$, les chaînes autoduales d'isogénies

$$
M_{V^{\prime}} \longrightarrow M_{V^{\prime}} / H \longrightarrow M_{V^{\prime}} / H^{\perp} \longrightarrow M_{V^{\prime}}
$$

dont la composée est la multiplication par $p^{n}$ et où $H \subset M_{V^{\prime}}\left[p^{n}\right]$ est un sous-groupe totalement isotrope qui relève $\left(V / V^{\prime \perp}\right) \otimes \mathbb{Z} / p^{n} \mathbb{Z}$.

On peut le décrire ainsi (voir $[\mathbf{F C}]$, Chap IV, sect. 6.5). C'est l'espace des trivialisations bilinéaires symétriques au dessus de $\frac{1}{p^{n}} V / V^{\prime \perp} \times \frac{1}{p^{n}} V / V^{\prime \perp}$ du fibré

$$
\left(c_{n} \times c_{n}\right)^{\star} \mathcal{P}^{-p^{n}} \text {. }
$$


On peut le munir d'une structure de torseur au dessus de $\mathfrak{B}_{V^{\prime}, n}$ sous le tore formel $p$-adique

$$
\operatorname{Hom}\left(\frac{1}{p^{n}} \operatorname{Sym}^{2}\left(V / V^{\prime \perp}\right), \widehat{\mathbb{G}}_{m}\right) .
$$

En effet, pour tout $\lambda \in \frac{1}{p^{n}} \operatorname{Sym}^{2}\left(V / V^{\prime \perp}\right)$, notons $\mathcal{L}(\lambda)$ le faisceau inversible sur $\mathfrak{B}_{V^{\prime}, n}$ obtenu en tirant en arrière le faisceau $\mathcal{P}^{p^{n}}$ le long de l'application $\mathfrak{B}_{V^{\prime}, n} \rightarrow A_{V^{\prime}} \times A_{V^{\prime}}$ obtenue en évaluant $c_{n} \times c_{n}$ sur $\frac{1}{p^{n}} \lambda$. On a des isomorphismes canoniques $\mathcal{L}\left(\lambda+\lambda^{\prime}\right)=$ $\mathcal{L}(\lambda) \otimes \mathcal{L}\left(\lambda^{\prime}\right)$ et $\mathfrak{M}_{V^{\prime}, n}$ n'est autre que le spectre formel relatif de l'algèbre gradué

$$
\mathfrak{M}_{V^{\prime}, n}=\operatorname{Spf}_{\mathfrak{B}_{V^{\prime}, n}}\left(\widehat{\bigoplus}_{\lambda} \mathcal{L}(\lambda)\right)
$$

où le chapeau désigne la complétion $p$-adique. C'est bien un torseur sous

$$
\operatorname{Hom}\left(\frac{1}{p^{n}} \operatorname{Sym}^{2}\left(V / V^{\prime \perp}\right), \widehat{\mathbb{G}}_{m}\right) \text {. }
$$

Remarquons bien qu'au dessus de $\mathfrak{M}_{V^{\prime}, n}$ on a une isogénie

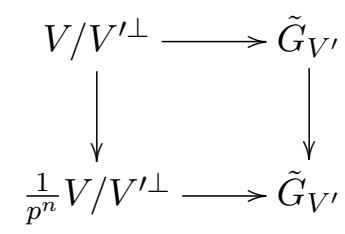

Son noyau vaut $V / V^{\prime \perp} \otimes \mathbb{Z} / p^{n} \mathbb{Z} \hookrightarrow M_{V^{\prime}}\left[p^{n}\right]$ et on dispose donc d'un isomorphisme

$$
M_{V^{\prime}}\left[p^{n}\right]=\left(V^{\prime} / p^{n} V^{\prime} \otimes \mu_{p^{n}}\right) \oplus A_{V^{\prime}}\left[p^{n}\right] \oplus\left(V / V^{\prime \perp} \otimes \mathbb{Z} / p^{n} \mathbb{Z}\right)
$$

qui explique pourquoi $\mathfrak{M}_{V^{\prime}, n}$ intervient dans la construction des compactifications toroïdales de niveau $p^{n}$.

- $\mathfrak{M}_{V^{\prime}, n} \rightarrow \mathfrak{M}_{V^{\prime}, n, \sigma}$ est le plongement torique affine formel associé au cône $\sigma \in C\left(V / V^{\prime \perp}\right)$

$$
\mathfrak{M}_{V^{\prime}, n, \sigma}=\operatorname{Spf}_{\mathcal{B}_{V^{\prime}, n}}\left(\widehat{\bigoplus}_{\lambda,<\lambda, \sigma>\geq 0} \mathcal{L}(\lambda)\right) .
$$

- $\mathfrak{M}_{V^{\prime}, n} \rightarrow \mathfrak{M}_{V^{\prime}, n, \mathcal{S}}$ est le plongement torique localement de type fini associé à la décomposition polyhédrale $\mathcal{S}$. Le schéma formel $\mathfrak{M}_{V^{\prime}, n, \sigma}$ est ouvert affine dans $\mathfrak{M}_{V^{\prime}, n, \mathcal{S}}$.

On note $\mathfrak{Z}_{n, \sigma}$ la strate fermée de $\mathfrak{M}_{V^{\prime}, n, \sigma}$ et $\mathfrak{Z}_{V^{\prime}, n}$ la strate fermée dans $\mathfrak{M}_{V^{\prime}, n, \mathcal{S}}$. Soit enfin $\Gamma_{V^{\prime}}$ le stabilisateur de $V^{\prime}$ dans $\Gamma$, qui agit sur

$$
C\left(V / V^{\prime \perp}\right)
$$

et sur le plongement torique $\mathfrak{M}_{V^{\prime}, n, \mathcal{S}}$.

A.2. Changement de niveau. - Pour tout $n \geq m$ on dispose d'un morphisme canonique $\mathfrak{X}_{V^{\prime}}\left(p^{n}\right) \rightarrow \mathfrak{X}_{V^{\prime}}\left(p^{m}\right)$ d'oubli partiel du niveau. On dispose également d'un morphisme $\mathfrak{B}_{V^{\prime}, n} \rightarrow \mathfrak{B}_{V^{\prime}, m}$ qui par les identifications

$$
\begin{aligned}
\mathfrak{B}_{V^{\prime}, n} & =\operatorname{Hom}_{\mathfrak{X}_{V^{\prime}}\left(p^{n}\right)}\left(\frac{1}{p^{n}} V / V^{\prime \perp}, A_{V^{\prime}}\right) \\
\mathfrak{B}_{V^{\prime}, m} & =\operatorname{Hom}_{\mathfrak{X}_{V^{\prime}}\left(p^{m}\right)}\left(\frac{1}{p^{m}} V / V^{\prime \perp}, A_{V^{\prime}}\right)
\end{aligned}
$$


correspond à l'inclusion $\frac{1}{p^{m}} V / V^{\perp} \subset \frac{1}{p^{n}} V / V^{\perp}$. Par les identifications précédentes

$$
\begin{aligned}
\mathfrak{B}_{V^{\prime}, n} & \simeq A_{V^{\prime}}^{r} \times \mathfrak{X}_{V^{\prime}} \mathfrak{X}_{V^{\prime}}\left(p^{n}\right) \\
\mathfrak{B}_{V^{\prime}, m} & \simeq A_{V^{\prime}}^{r} \times_{\mathfrak{X}_{V^{\prime}}} \mathfrak{X}_{V^{\prime}}\left(p^{m}\right)
\end{aligned}
$$

le morphisme $\mathfrak{B}_{V^{\prime}, n} \rightarrow \mathfrak{B}_{V^{\prime}, m} \times_{\mathfrak{X}_{V^{\prime}}\left(p^{m}\right)} \mathfrak{X}_{V^{\prime}}\left(p^{n}\right)$ correspond à la multiplication par $p^{n-m}$ de $A_{V^{\prime}}^{r}$. On dispose enfin d'un morphisme $\mathfrak{M}_{V^{\prime}, n, \sigma} \rightarrow \mathfrak{M}_{V^{\prime}, m, \sigma}$. Via les identifications

$$
\begin{aligned}
\mathfrak{M}_{V^{\prime}, n, \sigma} & =\operatorname{Spf}_{\mathcal{B}_{V^{\prime}, n}}\left(\widehat{\bigoplus}_{\lambda \in \frac{1}{p^{n}} \operatorname{Sym}^{2}\left(V / V^{\prime \perp}\right),<\lambda, \sigma>\geq 0} \mathcal{L}(\lambda)\right) \\
\mathfrak{M}_{V^{\prime}, m, \sigma} & =\operatorname{Spf}_{\mathcal{B}_{V^{\prime}, m}}\left(\widehat{\bigoplus}_{\lambda \in \frac{1}{p^{m}} \operatorname{Sym}^{2}\left(V / V^{\prime \perp}\right),<\lambda, \sigma>\geq 0} \mathcal{L}(\lambda)\right)
\end{aligned}
$$

il correspond à l'inclusion $\frac{1}{p^{m}} \operatorname{Sym}^{2}\left(V / V^{\perp}\right) \subset \frac{1}{p^{n}} \operatorname{Sym}^{2}\left(V / V^{\prime \perp}\right)$.

A.3. Compactification toroïdale. - On rappelle que $\mathfrak{X}$ est la variété de Siegel formelle de genre $g$ sur $\operatorname{Spf}\left(\mathcal{O}_{\mathbb{C}_{p}}\right)$ et de niveau premier à $p$ et que $\mathfrak{X}^{\text {tor }}$ est la compactification toroïdale associé à $\mathcal{S}$. Nous notons $\mathcal{X}\left(p^{n}\right)$ la variété rigide, finie étale sur $\mathcal{X}$ qui paramètre une structure pleine de niveau $p^{n}$ et $\mathcal{X}\left(p^{n}\right)^{\text {tor }}$ la compactification toroïdale associée à $\mathcal{S}$. On a donc un morphisme fini $\mathcal{X}\left(p^{n}\right)^{\text {tor }} \rightarrow \mathcal{X}^{\text {tor }}$ et nous notons $\mathfrak{X}\left(p^{n}\right)^{\text {tor }}$ la normalisation de $\mathfrak{X}^{\text {tor }}$ dans $\mathcal{X}\left(p^{n}\right)^{\text {tor }}$. La base donnée de $V$ fournit des structures principales de niveau $p^{n}$

$$
\psi_{n}:\left(\mathbb{Z} / p^{n} \mathbb{Z}\right)^{2 g} \simeq V / p^{n} V .
$$

Soit $\Gamma\left(p^{n}\right)$ le sous-groupe de congruence de $\Gamma=\mathrm{GSp}(V) \simeq \operatorname{GSp}_{2 g}(\mathbb{Z})$ qui laisse stable $\psi_{n}$ et soit $\Gamma\left(p^{n}\right)_{V^{\prime}}$ le stabilisateur de $V^{\prime}$ dans $\Gamma\left(p^{n}\right)$.

Théorème A.4. - Les points suivants sont vérifiés

1. La compactification torö̈dale $\mathfrak{X}\left(p^{n}\right)^{\text {tor }}$ possède une stratification indexée par $\mathfrak{C} / \Gamma\left(p^{n}\right)$. Pour tout $V^{\prime} \in \mathfrak{C}$, la complétion de $\mathfrak{X}\left(p^{n}\right)^{\text {tor }}$ le long de la $V^{\prime}$-strate est isomorphe à $\widehat{\mathfrak{M}}_{V^{\prime}, n, \mathcal{S}} / \Gamma\left(p^{n}\right)_{V^{\prime}}$ où $\widehat{\mathfrak{M}}_{V^{\prime}, n, \mathcal{S}}$ est la complétion de $\mathfrak{M}_{V^{\prime}, n, \mathcal{S}}$ le long de la strate $\mathfrak{Z}_{V^{\prime}, n}$.

2. La compactification torö̈dale $\mathfrak{X}\left(p^{n}\right)^{\text {tor }}$ possède une stratification plus fine indexée par $\mathcal{S} / \Gamma\left(p^{n}\right)$. Soit $\sigma \in \mathcal{S}$. La strate correspondante dans $\mathfrak{X}\left(p^{n}\right)^{\text {tor }}$ est isomorphe à $\mathfrak{Z}_{n, \sigma}$. Soit $\mathfrak{Z}$ un ouvert affine de $\mathfrak{Z}_{n, \sigma}$. L'hensélisation de $\mathfrak{X}\left(p^{n}\right)^{\text {tor }}$ le long de $\mathfrak{Z}$, notée $\mathfrak{X}\left(p^{n}\right)^{\text {tor,h, }}$, est isomorphe à l'hensélisation de $\mathfrak{M}_{V^{\prime}, n, \sigma}$ le long de $\mathfrak{Z}$, notée $\mathfrak{M}_{V^{\prime}, n, \sigma}^{h, \mathfrak{Z}}$.

3. Sur $\mathfrak{X}\left(p^{n}\right)^{\text {tor, } h, \mathfrak{Z}}$ on dispose par restriction du schéma semi-abélien A qui vérifie les compatibilités suivantes

- Sur l'ouvert de $\mathfrak{X}\left(p^{n}\right)^{\text {tor,h, } \mathfrak{Z}}$ où A est abélien, on a un isomorphisme

$$
M_{V^{\prime}}\left[p^{n}\right] \simeq A\left[p^{n}\right]
$$

Cet isomorphisme est compatible à la structure de niveau donné à gauche par l'égalité A.1.B et à droite par la structure universelle, la compatibilité étant fournie par l'isomorphisme

$$
\left(\mathbb{Z} / p^{n} \mathbb{Z}\right)^{2 g}=V / p^{n} V=V^{\prime} / p^{n} V^{\prime} \oplus\left(V^{\perp} / V^{\prime} \otimes \mathbb{Z} / p^{n}\right) \oplus\left(V / V^{\prime \perp} \otimes \mathbb{Z} / p^{n}\right) .
$$

- On a un isomorphisme $\omega_{A}=\omega_{\tilde{G}_{V^{\prime}}}$.

- On a un plongement $\tilde{G}\left[p^{n}\right] \hookrightarrow A$.

4. Les isomorphismes apparaissant dans les points précédents du théorème sont compatibles au morphisme canonique $\mathfrak{X}\left(p^{n}\right)^{\text {tor }} \rightarrow \mathfrak{X}\left(p^{m}\right)^{\text {tor }}$ pour tout $n \geq m$ et au morphisme décrit dans le paragraphe A.2. 
Démonstration. Lorsque $n=0$ ou au niveau rigide, c'est $[\mathbf{F C}]$, chap IV, sect. 5 et 6 . Comme la normalisation commute à la localisation étale, on se ramène à vérifier que nos cartes formelles de niveau $n$ sont la normalisation des cartes sans niveau, ce qui est évident. Pour l'avant dernier point voir $[\mathbf{F C}]$, Chap III, sect. 5 et également $[\mathbf{S t}]$, sect. 2.3.3. Le dernier point est clair.

A.5. Compactification minimale. - On suppose ici que $p^{n} \geq 3$ pour que le niveau soit net. C'est le cas pour tout $p$ si $n \geq n_{0}$ décrit précedemment. On rappelle que $\mathfrak{X}^{*}$ est la compactification minimale formelle sans niveau en $p$, que $\mathcal{X}\left(p^{n}\right)^{*}$ est la compactification minimale rigide de niveau plein $p^{n}$ et que $\mathfrak{X}\left(p^{n}\right)^{*}$ est la normalisée de $\mathfrak{X}^{*}$ dans $\mathcal{X}\left(p^{n}\right)^{*}$. On dispose de plus d'un morphisme canonique $f: \mathfrak{X}\left(p^{n}\right)^{\text {tor }} \rightarrow \mathfrak{X}\left(p^{n}\right)^{*}$ tel que $f_{*}(\mathcal{O})=\mathcal{O}$ et qui identifie $\mathfrak{X}\left(p^{n}\right)^{*}$ à la factorisation de Stein du faisceau semi-ample $\operatorname{det} \omega_{A}$ sur $\mathfrak{X}\left(p^{n}\right)^{\text {tor }}$.

Lemme A.6. - La compactification minimale $\mathfrak{X}\left(p^{n}\right)^{*}$ possède une stratification paramétrée par $\mathfrak{C} / \Gamma\left(p^{n}\right)$. Le morphisme $f$ est compatible à cette stratification et à celle décrite dans le théorème A.4.

Démonstration. Le morphisme $f$ est surjectif par construction. Il s'agit de vérifier que deux strates distinctes de $\mathfrak{X}\left(p^{n}\right)^{\text {tor }}$ ont des images différentes par $f$. Supposons que $x$ est un point dans l'image de la strate associée à $V^{\prime} \in \mathfrak{C}$. Soit $C$ une courbe propre lisse connexe s'envoyant sur $\pi^{-1}(x)$. D'après [FC, prop.V.2.2] on trouve que la restriction de $A$ à $C$ est extension d'un schéma abélien isotrivial par un tore déployé. Soit $y$ un point de $C$. Sur l'image de $\mathfrak{X}\left(p^{n}\right)$ dans le complété de $\mathfrak{X}\left(p^{n}\right)^{\text {tor }}$ en $y$ on obtient un isomorphisme $A\left[p^{n}\right] \simeq$ $\left(\mathbb{Z} / p^{n} \mathbb{Z}\right)^{2 g}=V / p^{n} V$ et la filtration à trois crans de $A\left[p^{n}\right]$ fournie par la construction de Mumford fournit un $V^{\prime \prime} \in \mathfrak{C}$ qui est bien déterminé modulo $\Gamma\left(p^{n}\right)$. L'application $y \mapsto V^{\prime \prime}$ de $C$ dans $\mathfrak{C} / \Gamma\left(p^{n}\right)$ ainsi obtenue est localement constante donc constante puisque $C$ est connexe. Elle est donc constante dans les fibres connexes de $\pi$. Ainsi $V^{\prime \prime}=V^{\prime}$ car cette égalité a lieu lorsque $y$ est dans la $V^{\prime}$-strate de $\mathfrak{X}\left(p^{n}\right)^{\text {tor }}$ par le point 3 du théorème A.4. Ainsi $V^{\prime} \in \mathfrak{C} / \Gamma\left(p^{n}\right)$ est uniquement déterminé par $x$, d'où le résultat.

Introduisons maintenant le développement de Fourier-Jacobi des sections de $\operatorname{det} \omega_{A}^{k}$ sur $\mathfrak{X}\left(p^{n}\right)^{\text {tor }}$ pour tout entier $k \geq 0$. Soit $V^{\prime} \in \mathfrak{C}$ et $\sigma \in \mathcal{S}$ qui est dans l'intérieur de $C\left(V / V^{\prime \perp}\right)$. Notons $\sigma^{\vee}$ l'ensemble des $\lambda$ tels que $\langle\lambda, \sigma\rangle \geq 0$ et $\sigma^{>}$l'ensemble des $\lambda$

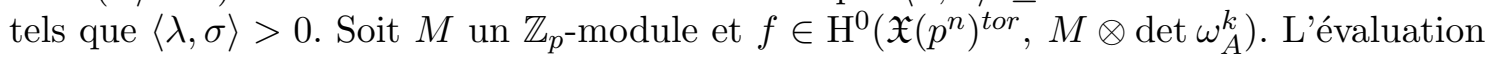
de $f$ sur le complété formel de $\mathfrak{M}_{V^{\prime}, n, \sigma}$ le long de la $\sigma$-strate fournit un élément

$$
\begin{gathered}
F J_{\sigma}(f)=\sum_{\lambda \in \frac{1}{p^{n}} \operatorname{Sym}^{2}\left(V / V^{\prime \perp}\right) \cap \sigma^{\vee}} a_{\lambda, \sigma}(f) \in \\
\prod_{\lambda \in \frac{1}{p^{n}} \operatorname{Sym}^{2}\left(V / V^{\prime \perp}\right) \cap \sigma^{\vee}} \mathrm{H}^{0}\left(\mathfrak{B}_{V^{\prime}, n}, \mathcal{L}(\lambda) \otimes M \otimes \operatorname{det}\left(\omega_{A_{V^{\prime}}} \otimes V / V^{\prime \perp}\right)^{k}\right) .
\end{gathered}
$$

On vérifie que $a_{\lambda, \sigma}(f)$ est indépendant de $\sigma$ et de $\mathcal{S}$ et on le note $a_{\lambda, V^{\prime}}(f)$. La famille des $\left(a_{\lambda, V^{\prime}}(f)\right)_{\lambda}$ est à support dans $\left.C\left(V / V^{\prime \perp}\right)^{\vee}\right)$.

Lemme A.7. - La restriction de $f$ à la $\sigma$-strate de $\mathfrak{X}\left(p^{n}\right)^{\text {tor }}$ ne dépend que de $V^{\prime}$ et est égale à

$a_{0, V^{\prime}}(f) \in \mathrm{H}^{0}\left(\mathfrak{B}_{V^{\prime}, n}, M \otimes \operatorname{det}\left(\omega_{A_{V^{\prime}}} \otimes V / V^{\perp}\right)^{k}\right)=\mathrm{H}^{0}\left(\mathfrak{X}_{V^{\prime}}\left(p^{n}\right), M \otimes \operatorname{det}\left(\omega_{A_{V^{\prime}}} \otimes V / V^{\prime \perp}\right)^{k}\right)$

Démonstration. L'idéal de la $\sigma$-strate est engendré par les $\mathrm{H}^{0}\left(\mathfrak{B}_{V^{\prime}, n}, \mathcal{L}(\lambda)\right)$ où $\lambda \in$ $\frac{1}{p^{n}} \operatorname{Sym}^{2}\left(V / V^{\perp \perp}\right) \cap \sigma^{>}$et le développement de Fourier-Jacobi est à support dans $C\left(V / V^{\prime \perp}\right)^{\vee}$. Or $C\left(V / V^{\prime \perp}\right)^{\vee} \backslash \sigma^{>}=\{0\}$ car $\sigma$ est inclus dans l'intérieur de $C\left(V / V^{\perp \perp}\right)$. 
Lemme A.8. - Soit $V^{\prime} \in \mathfrak{C} / \Gamma\left(p^{n}\right)$. La restriction de $f$ à la $V^{\prime}$-strate $\mathfrak{Z}_{V^{\prime}, n}$ de $\mathfrak{X}\left(p^{n}\right)^{\text {tor }}$ se factorise via les morphismes du diagramme A.1.A en une application radicielle de $\mathfrak{X}_{V^{\prime}}\left(p^{n}\right)$ dans $f\left(\mathfrak{Z}_{V^{\prime}, n}\right)$.

Démonstration. Comme $\mathfrak{X}\left(p^{n}\right)^{*}$ est la factorisation de Stein d'une puissance de $\operatorname{det} \omega_{A}$ sur $\mathfrak{X}\left(p^{n}\right)^{\text {tor }}$, le morphisme $f$ est obtenu en évaluant des éléments de $\mathrm{H}^{0}\left(\mathfrak{X}\left(p^{n}\right)^{\text {tor }}\right.$, $\left.\operatorname{det} \omega_{A}^{k}\right)$ pour $k$ variable. Le fait que la restriction de $f$ à $\mathfrak{Z}_{V^{\prime}, n}$ se factorise par $\mathfrak{X}_{V^{\prime}}\left(p^{n}\right)$ provient alors du lemme A.7. Soit $x \in f\left(\mathfrak{Z}_{V^{\prime}, n}\right)$ et $C$ une courbe propre lisse connexe s'envoyant dans $\pi^{-1}(x)$. D'après [FC, prop.V.2.2], la partie abélienne de la restriction de $A$ à $C$ est isotriviale d'où le caractère radiciel de la factorisation de $f$.

Lemme A.9. - Soit $V^{\prime} \in \mathfrak{C}$ et $x$ un point de la $V^{\prime}$-strate de $\mathfrak{X}\left(p^{n}\right)^{*}$. On note toujours $x$ l'unique point de $\mathfrak{X}_{V^{\prime}}\left(p^{n}\right)$ fourni par le morphisme radiciel $\mathfrak{X}_{V^{\prime}}\left(p^{n}\right) \rightarrow f\left(\mathfrak{Z}_{V^{\prime}, n}\right)$. Il existe un isomorphisme canonique

$$
\widehat{\mathscr{O}}_{\mathfrak{X}\left(p^{n}\right)^{*}, x}=\left(\prod_{\lambda \in \frac{1}{p^{n}} \operatorname{Sym}^{2}\left(V / V^{\prime \perp}\right) \cap C\left(V / V^{\prime \perp}\right)^{\vee}} \mathrm{H}^{0}\left(\widehat{\mathfrak{B}}_{V^{\prime}, n, x}, \mathcal{L}(\lambda)\right)\right)^{\Gamma\left(p^{n}\right)_{V^{\prime}}}
$$

où $\widehat{\mathfrak{B}}_{V^{\prime}, n, x}$ désigne le complété formel de $\mathfrak{B}_{V^{\prime}, n}$ le long de la fibre en $x$ du morphisme $\mathfrak{B}_{V^{\prime}, n} \rightarrow \mathfrak{X}_{V^{\prime}}\left(p^{n}\right)$.

Démonstration. C'est le théorème des fonctions formelles appliqué au morphisme $f$ combiné avec la description du complété de $\mathfrak{X}\left(p^{n}\right)^{\text {tor }}$ le long de la $V^{\prime}$-strate fournie par le point 1 du théorème A.4.

Corollaire A.10. - On a un isomorphisme canonique $f_{*}(\mathscr{O}) / p^{k}=f_{*}\left(\mathscr{O} / p^{k}\right)$.

Démonstration. Par dévissage, on se ramène à supposer $k=1$. On teste l'isomorphisme sur les complétés en chaque point. Soit $x$ un point de $\mathfrak{X}\left(p^{n}\right)^{*} \times \operatorname{Spec}\left(\mathbb{F}_{p}\right)$. Comme $f_{*}(\mathscr{O})=$ $\mathscr{O}$, on trouve d'après le lemme A.9 que

$\mathrm{H}^{0}\left(\widehat{\mathscr{O}}_{\mathfrak{X}\left(p^{n}\right)^{*}, x}, f_{*}(\mathscr{O}) / p\right)=\left(\prod_{\lambda \in \frac{1}{p^{n}} \operatorname{Sym}^{2}\left(V / V^{\prime \perp}\right) \cap C\left(V / V^{\prime} \perp\right)^{\vee}} \mathrm{H}^{0}\left(\widehat{\mathfrak{B}}_{V^{\prime}, n, x}, \mathcal{L}(\lambda)\right)\right)^{\Gamma\left(p^{n}\right)_{V^{\prime}}} \otimes \mathbb{F}_{p}$.

Toujours d'après le théorème des fonctions formelles on a

$\mathrm{H}^{0}\left(\widehat{\mathscr{O}}_{\mathfrak{X}\left(p^{n}\right)^{*}, x}, f_{*}(\mathscr{O} / p)\right)=\left(\prod_{\lambda \in \frac{1}{p^{n}} \operatorname{Sym}^{2}\left(V / V^{\prime \perp}\right) \cap C\left(V / V^{\prime \perp}\right)^{\vee}} \mathrm{H}^{0}\left(\widehat{\mathfrak{B}}_{V^{\prime}, n, x} \otimes \mathbb{F}_{p}, \mathcal{L}(\lambda)\right)\right)^{\Gamma\left(p^{n}\right)_{V^{\prime}}}$

Pour tout $\lambda$, le faisceau inversible $\mathcal{L}(\lambda)$ sur $\mathfrak{B}_{V^{\prime}, n}=\operatorname{Hom}_{\mathfrak{X}_{V^{\prime}}\left(p^{n}\right)}\left(\frac{1}{p^{n}} V / V^{\prime \perp}, A_{V^{\prime}}\right)$ provient par image inverse d'un faisceau inversible $\mathcal{L}^{\prime}(\lambda) \operatorname{sur}^{\operatorname{Hom}_{\mathfrak{X}_{V^{\prime}}\left(p^{n}\right)}}\left(V_{\lambda}^{\prime}, A_{V^{\prime}}\right)$ où $V_{\lambda}^{\prime}$ est le facteur direct minimal de $\frac{1}{p^{n}} V / V^{\prime \perp}$ tel que $\lambda$ se factorise par le dual de $V_{\lambda}^{\prime}$. On obtient donc une surjection de schémas abéliens

$$
\operatorname{Hom}_{\mathfrak{X}_{V^{\prime}}\left(p^{n}\right)}\left(\frac{1}{p^{n}} V / V^{\prime \perp}, A_{V^{\prime}}\right) \longrightarrow \operatorname{Hom}_{\mathfrak{X}_{V^{\prime}}\left(p^{n}\right)}\left(V_{\lambda}^{\prime}, A_{V^{\prime}}\right)
$$

sur $\mathfrak{X}_{V^{\prime}}\left(p^{n}\right)$ et $\mathcal{L}^{\prime}(\lambda)$ est relativement ample. La formation de ses sections globales commute donc à la réduction modulo $p$ par le théorème de Riemann-Roch pour les schémas abéliens. De plus le stabilisateur de $\lambda$ dans $\Gamma\left(p^{n}\right)_{V^{\prime}}$ agit trivialement sur $V_{\lambda}^{\prime}$ donc la formation des invariants par $\Gamma\left(p^{n}\right)_{V^{\prime}}$ commute également à la réduction modulo $p$. 
En raisonnant comme [FC, th.V.2.5] il est alors aisé de démontrer le résultat suivant, que nous n'utiliserons pas.

Théorème A.11. - La compactification minimale $\mathfrak{X}\left(p^{n}\right)^{*}$ possède une stratification paramétrée par $\mathfrak{C} / \Gamma\left(p^{n}\right)$. La strate associée à $V^{\prime} \in \mathfrak{C}$ est canoniquement isomorphe à $\mathfrak{X}_{V^{\prime}}\left(p^{n}\right)$.

A.12. Compactification toroïdale perfectoïde. - Notons $\mathfrak{X}\left(p^{\infty}\right)^{\text {tor-mod }}$ la limite projective sur $n \geq n_{0}$ des $\mathfrak{X}\left(p^{n}\right)^{\text {tor-mod }}$ dans la catégorie des schémas formels $p$-adiques et $\mathcal{X}\left(p^{\infty}\right)^{\text {tor-mod }}$ sa fibre générique dans le sens de [SW]. Notre but dans ce paragraphe est de vérifier que $\mathcal{X}\left(p^{\infty}\right)^{\text {tor-mod }}$ est perfectoïde sachant que c'est le cas des variétés de Siegel ouvertes de niveau infini et de tout genre d'après $[\mathbf{S} 4]$.

Remarque A.13. - Soit $\mathfrak{X}\left(p^{\infty}\right)^{\text {tor }}$ la limite projective des $\mathfrak{X}\left(p^{n}\right)^{\text {tor }}$ dans la catégorie des schémas formels $p$-adiques et $\mathcal{X}\left(p^{\infty}\right)^{\text {tor }}$ sa fibre générique dans le sens de $[\mathbf{S W}]$. On dispose d'un morphisme $\mathcal{X}\left(p^{\infty}\right)^{\text {tor-mod }} \rightarrow \mathcal{X}\left(p^{\infty}\right)^{\text {tor }}$ mais nous ne savons pas si c'est un isomorphisme.

Soit $n \geq n_{0}$ et $V^{\prime} \in \mathfrak{C}$. On a introduit précédemment la variété de Siegel $\mathfrak{X}_{V^{\prime}}\left(p^{n}\right) \rightarrow$ $\operatorname{Spf}\left(\mathcal{O}_{\mathbb{C}_{p}}\right)$ de genre $g-r$ où $r=\operatorname{rg}_{\mathbb{Z}}\left(V^{\prime}\right)$. Elle admet également une compactification toroïdale $\mathfrak{X}_{V^{\prime}}\left(p^{n}\right)^{\text {tor }}$ construite par normalisation. On peut lui appliquer les constructions de la première partie en remplaçant partout $g$ par $g-r$ et l'on obtient un schéma formel admissible normal $\mathfrak{X}_{V^{\prime}}\left(p^{n}\right)^{\text {tor-mod }}$ muni d'une flèche vers $\mathfrak{X}_{V^{\prime}}\left(p^{n}\right)^{\text {tor }}$. On pose alors

$$
\begin{aligned}
\mathfrak{X}_{V^{\prime}}\left(p^{n}\right)^{\text {mod }} & =\mathfrak{X}_{V^{\prime}}\left(p^{n}\right) \times_{\mathfrak{X}_{V^{\prime}}\left(p^{n}\right)^{\text {tor }}} \mathfrak{X}_{V^{\prime}}\left(p^{n}\right)^{\text {tor }- \text { mod }}, \\
\mathfrak{B}_{V^{\prime}, n}^{\text {mod }} & =\mathfrak{B}_{V^{\prime}, n} \times_{\mathfrak{X}_{V^{\prime}}\left(p^{n}\right)} \mathfrak{X}_{V^{\prime}}\left(p^{n}\right)^{\text {mod }}, \\
\mathfrak{M}_{V^{\prime}, n, \sigma}^{\text {mod }} & =\mathfrak{M}_{V^{\prime}, n, \sigma} \times_{\mathfrak{X}_{V^{\prime}}\left(p^{n}\right)} \mathfrak{X}_{V^{\prime}}\left(p^{n}\right)^{\text {mod }}, \\
\mathfrak{Z}_{n, \sigma}^{\text {mod }} & =\mathfrak{Z}_{n, \sigma} \times_{\mathfrak{X}_{V^{\prime}}\left(p^{n}\right)} \mathfrak{X}_{V^{\prime}}\left(p^{n}\right)^{\text {mod }}
\end{aligned}
$$

pour tout $\sigma \in \mathcal{S}$ qui est inclus dans l'intérieur de $C\left(V / V^{\perp \perp}\right)$.

Soit $\hat{\sigma}=\left(\sigma_{n}\right) \in \lim _{n} \mathcal{S} / \Gamma\left(p^{n}\right)$ et soit $\hat{V}^{\prime}=\left(V_{n}^{\prime}\right) \in \lim _{n} \mathfrak{C} / \Gamma\left(p^{n}\right)$ tels que $\sigma_{n}$ soit dans l'intérieur de $V_{n}^{\prime}$, on peut former dans la catégorie des schémas formels $p$-adiques les limites projectives

$$
\begin{aligned}
\mathfrak{X}_{\hat{V}^{\prime}}\left(p^{\infty}\right)^{\text {mod }} & ={\underset{n}{\lim }}_{\mathfrak{X}_{V_{n}^{\prime}}\left(p^{n}\right)^{\text {mod }}} \\
\mathfrak{B}_{\hat{V}^{\prime}, \infty}^{\text {mod }} & ={\underset{n}{\lim } \mathfrak{B}_{V_{n}^{\prime}, n}^{\text {od }}}_{\mathfrak{M}_{\hat{V}^{\prime}, \infty, \hat{\sigma}}^{\text {mod }}}=\underset{\lim _{n}}{\mathfrak{M}_{V_{n}^{\prime}, n, \sigma_{n}}^{\text {mod }}} \\
\mathfrak{Z}_{\infty, \hat{\sigma}}^{\text {mod }} & ={\underset{n}{\lim }}_{\mathfrak{Z}_{n, \sigma_{n}}^{\text {mod }} .}
\end{aligned}
$$

On obtient la proposition suivante par changement de base du théorème A.4 par $\mathfrak{X}\left(p^{n}\right)^{\text {tor-mod }} \rightarrow \mathfrak{X}\left(p^{n}\right)^{\text {tor }}$ et passage à la limite.

Proposition A.14. - 1. Le schéma formel $\mathfrak{X}\left(p^{n}\right)^{\text {tor-mod }}$ possède une stratification indexée par $\mathcal{S} / \Gamma\left(p^{n}\right)$ et pour tout $\sigma \in \mathcal{S}$, la $\sigma$-strate associée est canoniquement isomorphe à $\mathfrak{Z}_{n, \sigma}^{\text {mod }}$.

2. La complétion formelle de $\mathfrak{X}\left(p^{n}\right)^{\text {tor-mod }}$ le long de la $\sigma$-strate est canoniquement isomorphe à la complétion formelle de $\mathfrak{M}_{V^{\prime}, n, \sigma}^{\text {mod }}$ le long de $\mathfrak{Z}_{n, \sigma}^{\text {mod }}$. Pour tout point géométrique $x$ de la $\sigma$-strate $\mathfrak{Z}_{n, \sigma}^{\text {mod }}$, les hensélisés stricts en $x$ de $\mathfrak{X}\left(p^{n}\right)^{\text {tor-mod }}$ et $\mathfrak{M}_{V^{\prime}, n, \sigma}^{\text {mod }}$ sont isomorphes. 
3. Le schéma formel $\mathfrak{X}\left(p^{\infty}\right)^{\text {tor-mod }}$ possède une stratification indexée par l'ensemble pro-fini $\lim _{n} \mathcal{S} / \Gamma\left(p^{n}\right)$. Soit $\hat{\sigma}=\left(\sigma_{n}\right)_{n \geq n_{0}} \in \lim _{n} \mathcal{S} / \Gamma\left(p^{n}\right)$ et $\hat{V}^{\prime}=\left(V_{n}^{\prime}\right)$ tel que $\sigma_{n}$ soit dans l'intérieur de $C\left(V / V_{n}^{\prime \perp}\right)$. La $\hat{\sigma}$-strate est canoniquement isomorphe à $\mathfrak{Z}_{\infty, \hat{\sigma}}^{\text {mod }}$ et la complétion formelle le long de la $\hat{\sigma}$-strate est isomorphe à la complétion formelle de $\mathfrak{M}_{\hat{V}^{\prime}, n, \hat{\sigma}}^{\text {mod }}$ le long de cette strate.

Lemme A.15. - Soit $C$ un corps non-archimédien complet algébriquement clos pour une valuation de rang $1, \mathcal{O}_{C}$ son anneau d'entiers et $\varpi$ une pseudo-uniformisante. Soit $\left(\mathfrak{X}_{n}\right)_{n \geq 0} \rightarrow \operatorname{Spf}\left(\mathcal{O}_{C}\right)$ un système projectif de schémas formels $\varpi$-adiques normaux, plats, topologiquement de type fini sur $\operatorname{Spf}\left(\mathcal{O}_{C}\right)$. On suppose les applications de transition finies et surjectives. Soit $\operatorname{Spf}\left(R_{0}\right) \hookrightarrow \mathfrak{X}_{0}$ un ouvert affine formel et $\operatorname{Spf}\left(R_{n}\right)$ son image inverse dans $\mathfrak{X}_{n}$ pour tout $n \geq 0$. Soit $R_{\infty}$ la complétion $\varpi$-adique de la limite inductive des $R_{n}$. La norme supremum sur $R_{k}$ pour tout $k$ induit une norme $|$.$| sur R_{\infty}$ et la topologie définie par cette norme est la topologie $\varpi$-adique. On a $\left|R_{\infty}[1 / \varpi]\right|=|C|$ et $R_{\infty}=\left\{x \in R_{\infty}[1 / \varpi],|x| \leq 1\right\}$.

Démonstration. La norme supremum sur $R_{k}$ pour tout $k$ induit bien une norme|.| sur $R_{\infty}$ et la topologie induite est bien la topologie $\varpi$-adique. Pour tout $n,\left|R_{k}\right|=\left|\mathcal{O}_{C}\right|$ d'après [BGR], sect. 6.4.3, thm 1 et coro. 6. Il en résulte que $\left|R_{\infty}\right|=\left|\mathcal{O}_{C}\right|$. Soit $R_{\infty}[1 / \varpi]^{0}$ l'ensemble des éléments à puissance bornée dans $R_{\infty}[1 / \varpi]$. L'inclusion de $R_{\infty}$ dans $R_{\infty}[1 / \varpi]^{0}$ est claire. Réciproquement soit $f \in R_{\infty}[1 / \varpi]^{0}$. Il existe alors une suite $f_{n} \in R_{n}[1 / \varpi]$ qui converge $\varpi$-adiquement vers $f$. Pour $n$ assez grand, $f_{n}$ est clairement à puissances bornées donc dans $R_{n}=R_{n}[1 / p]^{0}$ par normalité de $R_{n}$. On en déduit bien que $f \in R_{\infty}$.

Lemme A.16. - Soit $S$ un schéma formel p-adique normal et plat sur $\operatorname{Spf}\left(\mathcal{O}_{\mathbb{C}_{p}}\right)$ de fibre générique perfectoïde et soit $A$ un schéma abélien sur $S$. Notons

$$
\tilde{A}=\underset{p}{\lim } A
$$

où les flèches de transition sont la multiplication par p et la limite projective est prise dans la catégorie des schémas formels p-adiques. Notons $\tilde{\mathcal{A}}$ la fibre générique de $\tilde{A}$ dans le sens de $[\mathbf{S W}]$. Alors $\tilde{\mathcal{A}}$ est perfectoïde.

Démonstration. On se restreint au cas où $S=\operatorname{Spf} R_{0}$ est affine. Notons $R=R_{0}[1 / p]$ et $R^{0} \subset R$ les éléments de puissances bornées. On a $R^{0}=R_{0}$ par normalité. Fixons $p^{b} \in \mathcal{O}_{\mathbb{C}_{p}}^{b}$ tel que $\left(p^{b}\right)^{\sharp}=p$ et notons $R^{b}$ le tilt de $R$ sur $\mathbb{C}_{p}^{b}$. Notons $\bar{A} \rightarrow \operatorname{Spec}\left(R_{0} / p\right)$ la réduction de $A$ modulo $p$. Comme

$$
R_{0} / p=R^{0, b} / p^{b}
$$

on peut voir $\bar{A}$ comme un schéma abélien $\operatorname{sur} \operatorname{Spec}\left(R^{0, b} / p^{b}\right)$. La théorie des déformations montre que $\bar{A}$ se relève en un schéma abélien $A^{b} \operatorname{sur} \operatorname{Spf}\left(R^{0, b}\right)$. On peut alors former la limite projective

$$
\tilde{A}^{b}=\lim _{p} A^{b}
$$

dans la catégorie des schémas formels $p^{b}$-adiques $\operatorname{sur} \operatorname{Spf}\left(R^{0, b}\right)$.

Montrons que $\tilde{A}^{b}$ est parfait. Soit $F_{\text {abs }}: \tilde{A}^{b} \rightarrow \tilde{A}^{b}$ le morphisme de Frobenius absolu et $\left(\tilde{A}^{b}\right)^{(p)}$ le changement de base par le morphisme de Frobenius absolu $R^{0, b} \rightarrow R^{0, b}$. Le Frobenius absolu $F_{a b s}$ de $\tilde{A}^{b}$ s'écrit $F_{\text {abs }}=\varphi \circ F_{\text {rel }}$ où $F_{\text {rel }}: \tilde{A}^{b} \rightarrow\left(\tilde{A}^{b}\right)^{p}$ est le morphisme de Frobenius relatif et $\varphi:\left(\tilde{A}^{b}\right)^{(p)} \rightarrow \tilde{A}^{b}$ est le morphisme de changement de base au dessus du Frobenius absolu de $R^{0, b}$. D'après [S2, prop.5.9], $R^{0, b}$ est parfait et $\varphi$ est bijectif. Soit $V_{\text {rel }}:\left(\tilde{A}^{b}\right)^{(p)} \rightarrow \tilde{A}^{b}$ le morphisme de Verschiebung induit par celui de $A^{b}$. Posons 
$V_{\text {abs }}=V_{\text {rel }} \circ \varphi^{-1}$. Comme $V_{\text {rel }} \circ F_{\text {rel }}=F_{\text {rel }} \circ V_{\text {rel }}=p$, on a $V_{\text {abs }} \circ F_{\text {abs }}=F_{\text {abs }} \circ V_{\text {abs }}=p$. Mais la multiplication par $p$ est un automorphisme de $\tilde{A}^{b}$ donc il en est de même pour $F_{\text {abs }}$.

Montrons que la fibre générique $\tilde{\mathcal{A}}^{b}$ de $\tilde{A}^{b}$ dans le sens de $[\mathbf{S W}]$ est perfectoïde sur $\mathbb{C}_{p}^{b}$. Soit $\operatorname{Spf}\left(T_{0}\right)$ un ouvert affine de $\tilde{A}^{b}$ et $T=T_{0}\left[1 / p^{b}\right]$ qui est une algèbre parfaite. On a $T^{0}=T_{0}$ d'après le lemme A.15 et $T^{0}$ est donc ouvert borné dans $T$. On conclut grâce à $\left[\mathbf{S 2}\right.$, prop.5.9]. On en déduit finalement que $\tilde{\mathcal{A}}$ est perfectoïde sur $\mathbb{C}_{p}$.

Lemme A.17. - Soit $\hat{\sigma}=\left(\sigma_{n}\right) \in \lim _{n} \mathcal{S} / \Gamma\left(p^{n}\right)$ et soit $\hat{V}^{\prime}=\left(V_{n}^{\prime}\right) \in \lim _{n} \mathfrak{C} / \Gamma\left(p^{n}\right)$ tels que $\sigma_{n}$ soit dans l'intérieur de $V_{n}^{\prime}$. La fibre générique de $\mathfrak{M}_{\hat{V}^{\prime}, \infty, \hat{\sigma}}^{\text {mod }}$ dans le sens de $[\mathbf{S W}]$ est perfectoïde.

Démonstration. D'après [S4], la fibre génerique de $\mathfrak{X}_{\hat{V}^{\prime}}\left(p^{\infty}\right)^{\text {mod }}$ est perfectoïde (voir la démonstration du théorème 1.18). D'après la discussion du paragraphe A.2, le morphisme $\mathfrak{B}_{\hat{V}^{\prime}, \infty}^{\text {mod }} \rightarrow \mathfrak{X}_{V^{\prime}}\left(p^{\infty}\right)^{\text {mod }}$ s'identifie à

$$
\lim _{p} A^{r}
$$

où $A$ est la variété abélienne universelle de dimension $g-r$ sur $\mathfrak{X}_{\hat{V}^{\prime}}\left(p^{\infty}\right)^{\text {mod }}$ (et $r$ est le rang de $V_{n}^{\prime}$ pour tout $n$ ). D'après le lemme A.16, la fibre générique de $\mathfrak{B}_{V^{\prime}, \infty}^{\text {mod }}$ est perfectoïde. Toujours d'après le paragraphe A.2, on a un isomorphisme

$$
\mathfrak{M}_{\hat{V}^{\prime}, \infty, \hat{\sigma}}^{\text {mod }}=\operatorname{Spf}_{\mathcal{B}_{V^{\prime}, \infty}^{\text {mod }}}\left(\widehat{\bigoplus}_{\lambda \in \operatorname{colim}_{n} \operatorname{Sym}^{2}\left(V / V_{n}^{\prime \perp}\right) \otimes_{\mathbb{Z}} \mathbb{Z}\left[1 / p^{n}\right],<\lambda, \sigma_{n}>\geq 0} \mathcal{L}(\lambda)\right)
$$

duquel on déduit finalement que la fibre générique de $\mathfrak{M}_{V^{\prime}, \infty, \sigma}^{\text {od }}$ est perfectoïde : c'est la perfectoïsation d'un plongement torique affine.

Proposition A.18. - Le Frobenius absolu induit une surjection

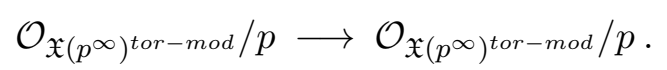

Démonstration. Notons $f_{n}: \mathfrak{X}\left(p^{n}\right)^{\text {tor-mod }} \rightarrow \mathfrak{X}\left(p^{n_{0}}\right)^{\text {tor-mod }}$ l' application de transition. Soit $x$ un point géométrique de $\mathfrak{X}\left(p^{n_{0}}\right)^{\text {tor-mod }}$ et $R_{n_{0}}$ l'hensélisé strict de $\mathscr{O}_{\mathfrak{X}\left(p^{n_{0}}\right)^{\text {tor-mod }}} / p$ en $x$. Notons $\operatorname{Spec}\left(R_{n}\right)$ l'image inverse de $\operatorname{Spec}\left(R_{n_{0}}\right)$ dans $\mathfrak{X}\left(p^{n}\right)^{\text {tor-mod }}$ pour $n \geq n_{0}$. Il suffit de montrer que le Frobenius absolu de $R_{\infty}=\operatorname{colim}_{n} R_{n}$ est surjectif.

Le morphisme $R_{n_{0}} \rightarrow R_{n}$ est fini et identifie $R_{n}$ à la somme directe des hensélisés

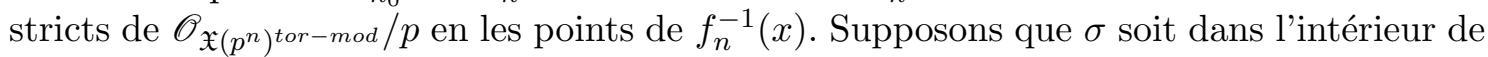
$C\left(V / V_{n_{0}}^{\perp \perp}\right)$ On peut identifier $R_{n_{0}}$ à l'hensélisé strict de $\mathfrak{M}_{V_{n_{0}}^{\prime}, n_{0}, \sigma}^{\text {mod }}$ en $x$ d'après la proposition A.14. Il en résulte que $R_{\infty}$ s'identifie à :

$$
\left(\operatorname{colim}_{\sigma_{n}, V_{n}^{\prime}} \mathscr{O}_{\mathfrak{M}_{V_{n}^{\prime}, n, \sigma_{n}}^{m o d}}\right) \otimes_{\mathscr{O}_{\mathfrak{M}_{V_{n_{0}}^{\prime}, n_{0}, \sigma}^{m o d}}} R_{0}
$$

où $\sigma_{n} \in \mathcal{S} / \Gamma\left(p^{n}\right)$ et $V_{n}^{\prime} \in \mathfrak{C} / \Gamma\left(p^{n}\right)$ est tel que $\sigma_{n}$ soit dans l'intérieur de $C\left(V / V_{n}^{\prime \perp}\right)$ et où $\sigma_{n}$ et $V_{n}^{\prime}$ sont congrus à $\sigma$ et $V_{n_{0}}$ modulo $p^{n_{0}}$. Pour $m \geq n$ et $\sigma_{m}$ d'image $\sigma_{n}$ dans $\mathcal{S} / \Gamma\left(p^{n}\right)$, on dispose d'une application naturelle

$$
\mathfrak{M}_{V_{m}^{\prime}, m, \sigma_{m}}^{\text {mod }} \rightarrow \mathfrak{M}_{V_{n}^{\prime}, n, \sigma_{n}}^{\text {mod }}
$$

qui induit les applications de transition dans la limite inductive précédente. Le faisceau

est un quotient du faisceau

$$
\operatorname{colim}_{\sigma_{n}, V_{n}^{\prime}} \mathscr{O}_{\mathfrak{M}_{V_{n}^{\prime}, n, \sigma_{n}}^{\text {mod }}} / p
$$

$$
\bigoplus_{\hat{\sigma} \in \lim _{n} \mathcal{S} / \Gamma\left(p^{n}\right)} \mathscr{O}_{\mathfrak{M}_{V^{\prime}, \infty, \hat{\sigma}}^{\text {mod }}} / p
$$


Le lemme A.17 permet donc de conclure.

Corollaire A.19. - L'espace $\mathcal{X}\left(p^{\infty}\right)^{\text {tor-mod }}$ est perfectö̈de.

Démonstration. Soit $\operatorname{Spf}\left(R_{\infty}\right) \subset \mathfrak{X}\left(p^{\infty}\right)^{\text {tor-mod }}$ un ouvert formel provenant de $\operatorname{Spf}\left(R_{0}\right) \subset$ $\mathfrak{X}\left(p^{n_{0}}\right)^{\text {tor-mod }}$ comme dans le lemme A.15. Alors $R_{\infty}[1 / p]^{0}=R_{\infty}$ est ouvert borné par construction de la topologie de $R_{\infty}[1 / p]$. Le Frobenius modulo $p$ est surjectif d'après la proposition A.18.

\section{Références}

[AIP] F. Andreatta, A. Iovita, V. Pilloni, p-adic families of Siegel modular cuspforms, à paraître à Annals of Maths.

[A] J. Arthur, The endoscopic classification of representations : orthogonal and symplectic groups, Colloquium Publications of the American Mathematical Society, volume 61.

[BGR] S. Bosch, U. Guntzer et R. Remmert, Non-Archimedean analysis, volume 261 of Grundlehren der Mathematischen Wissenschaften, Springer-Verlag, Berlin, 1984.

[D] P. Deligne, Travaux de Shimura, Séminaire Bourbaki 389 (1971).

[DM] P. Deligne et J. Milne, Tannakian categories, dans "Hodge Cycles, Motives, and Shimura Varieties", LNM 900 (1982), 101-228.

[F] L. Fargues, La filtration de Harder-Narasimhan des schémas en groupes finis et plats, J. Reine Angew. Math. 645 (2010), 1-39.

[FC] G. Faltings, C.-L. Chai, Degeneration of abelian varieties, Ergebnisse der Mathematik und ihrer Grenzbgebiete (3), vol. 22, Springer-Verlag, Berlin, (1990).

[G] W. Goldring, Galois representations associated to holomorphic limits of discrete series. I. Unitary groups, à paraître à Compositio.

[GN] W. Goldring et M.H. Nicole, The $\mu$-ordinary Hasse invariant of unitary Shimura varieties, prépublication.

[H1] M. Harris, Automorphic forms of $\bar{\partial}$-cohomology type as coherent cohomology classes, J. Diff. Geom., 32 (1990), p. 1-63.

[H2] M. Harris, Functorial properties of toroidal compactifications of locally symmetric varieties, Proc. London Math. Soc. (3) 59 (1989), p. 1-22.

[H3] M. Harris, Automorphic forms and the cohomology of vector bundles on Shimura varieties, Automorphic forms, Shimura varieties and $L$-functions, vol. 2, Academic press, p. 41-91.

[KMSW] T. Kaletha, A. Minguez, S.W. Shin, P.J. White, Endoscopic Classification of Representations : Inner Forms of Unitary Groups, prépublication (2014).

[Ki] M. Kisin, Integral models for Shimura varieties of abelian type, J. Amer. Math. Soc. 23 (4) (2010), p. 967-1012.

[Ko] R. E. Kottwitz, Points on some Shimura varieties over finite fields, J. Amer. Math. Soc. 5 (2) (1992), p. 373-444.

[L1] K.W. Lan, Arithmetic compactifications of PEL-type Shimura varieties, London Mathematical Society Monographs, vol. 36, Princeton University Press, Princeton (2013).

[LSt] K.W. Lan et B. Stroh, Relative cohomology of cuspidal forms on PEL type Shimura varieties, prépublication.

[LSu] K.W. Lan et J. Suh, Vanishing theorems for torsion automorphic sheaves on general PEL-type Shimura varieties, Adv. Math. 242 (2013), p. 228-286.

[LSw] J.S. Li et J. Schwermer, On the Eisenstein cohomology of arithmetic groups, Duke Math. J. 123 (2004), no. 1, p.141-169. 
[L2] G. Laumon, Fonctions zétas des variétés de Siegel de dimension trois, Formes automorphes. II. Le cas du groupe GSp(4), Astérisque no. 302 (2005), p.1-66.

[Ma] K. Madapusi Pera, Toroidal compactifications of integral models of Shimura varieties of Hodge type, prépublication (2014).

[Mi] J. Milne, Canonical models of (mixed) Shimura varieties and automorphic vector bundles, Automorphic forms, Shimura varieties and $L$-functions, vol. 1, Academic press, p. 283415.

[Mo] C.P. Mok, Endoscopic classification of representations of quasi-split unitary groups, à paraître dans Memoirs of the American Mathematical Society.

[P] R. Pink, Arithmetical compactification of mixed Shimura varieties, thèse de doctorat.

[Rap] M. Rapoport, Compactifications de l'espace de modules de Hilbert-Blumenthal, Compositio Math. 36 (1978), 255 -335.

[S1] P. Scholze, The Langlands-Kottwitz method and deformation spaces of p-divisible groups, J. Amer. Math. Soc. 26 (2013), no. 1, 227-259.

[S2] P. Scholze, Perfectoid spaces, Publ. math. de l'IHES 116 (2012), no. 1, 245-313.

[S3] P. Scholze, p-adic Hodge theory for rigid analytic varieties, Forum of Mathematics, Pi 1, e1 (2013).

[S4] P. Scholze, On torsion in the cohomology of locally symmetric varieties, prépublication.

[SW] P. Scholze et J. Weinstein, Moduli of p-divisible groups, à paraître dans Cambridge Journal of Mathematics.

[T] R. Taylor, On the $\ell$-adic cohomology of Siegel threefolds, Inventiones 114 (2) (1993), p.289-310.

[St] B.Stroh, Compactification de variétés de Siegel aux places de mauvaise réduction, Bull. Soc. Math. France 138 (2010), no. 2, p. 259-315.

[W] R. Weissauer, Four dimensional Galois representations, Formes automorphes. II. Le cas du groupe GSp(4), Astérisque no. 302 (2005), p.67-150

[X] B. Xu, Endoscopic classification of representations of $\operatorname{GSp}(2 n)$ and $\operatorname{GSO}(2 n)$, thèse de l'Université de Toronto, 2014.

Vincent Pilloni et Benoît Stroh 